\title{
SUBGRID UPSCALING AND MIXED MULTISCALE FINITE ELEMENTS*
}

\author{
TODD ARBOGAST ${ }^{\dagger}$ AND KIRSTEN J. BOYD
}

\begin{abstract}
Second order elliptic problems in divergence form with a highly varying leading order coefficient on the scale $\epsilon$ can be approximated on coarse meshes of spacing $H \gg \epsilon$ only if one uses special techniques. The mixed variational multiscale method, also called subgrid upscaling, can be used, and this method is extended to allow oversampling of the local subgrid problems. The method is shown to be equivalent to the multiscale finite element method when one uses the lowest order Raviart-Thomas spaces and provided that there are no fine scale components in the source function $f$. In the periodic setting, a multiscale error analysis based on homogenization theory of the more general subgrid upscaling method shows that the error is $O\left(\epsilon+H^{m}+\sqrt{\epsilon / H}\right)$, where $m=1$. Moreover, $m=2$ if one uses the second order Brezzi-Douglas-Marini or Brezzi-Douglas-Durán-Fortin spaces and no oversampling. The error bounding constant depends only on the $H^{m-1}$-norm of $f$ and so is independent of small scales when $m=1$. When oversampling is not used, a superconvergence result for the pressure approximation is shown.
\end{abstract}

Key words. mixed method, multiscale finite element, subgrid upscaling, variational multiscale

AMS subject classifications. 65N15, 65N30, 35J20

DOI. $10.1137 / 050631811$

1. Introduction. Many physical problems can be modeled by a second order elliptic partial differential equation in space. In many cases, the coefficients of the equation are highly heterogeneous, which induces fine scale variability in the solution. Thus the difficulty in approximating the solution on a coarse finite element mesh $\mathcal{T}_{H}$ is that the solution is not fully resolved on this scale. Traditional finite element analysis fails, and we require some multiscale approximation techniques.

Babuška and Osborn $[10,9]$ proposed using special finite elements to approximate the solution. Hughes et al. [23, 24] (see also [13]) developed a more formal framework, which they called the variational multiscale method. A mixed variant, described as subgrid upscaling, was developed by Arbogast et al. [7, 3, 4, 6, 5]. Hou and Wu [21] and $\mathrm{Hou}, \mathrm{Wu}$, and Cai [22] took a more direct approach and simply proposed finding a special finite element basis by solving the problem locally. They called this approach the multiscale finite element method. A mixed form was developed later by Chen and Hou [17].

To be more precise, consider a connected, convex polygonal domain $\Omega \subseteq \mathbb{R}^{d}$, where $d=2$ or 3 , and a second order, uniformly positive-definite symmetric tensor $a$, so that both $a$ and $a^{-1}$ are uniformly elliptic and uniformly bounded. Suppose we are also given vectors $\mathbf{b}$ and $\mathbf{v}_{g}$. For a set $S$, let $\nu^{S}$ be the outward unit normal to $\partial S$, and define the function $g$ on $\partial \Omega$ by $g=\mathbf{v}_{g} \cdot \nu$, where $\nu=\nu^{\Omega}$. The problem under

*Received by the editors May 18, 2005; accepted for publication (in revised form) February 3, 2006; published electronically June 21, 2006.

http://www.siam.org/journals/sinum/44-3/63181.html

${ }^{\dagger}$ Department of Mathematics, University of Texas, 1 University Station C1200, Austin, TX 78712, and Institute for Computational Engineering and Sciences, University of Texas, 1 University Station C0200, Austin, TX 78712 (arbogast@ices.utexas.edu). The work of this author was supported by the U.S. National Science Foundation under grant DMS-0408489.

${ }^{\ddagger}$ Eureka College, 300 E. College Ave., Eureka, IL 61530 (kboyd@eureka.edu). 
consideration is to find $\mathbf{u}$ and $p$ such that

$$
\begin{array}{ll}
\nabla \cdot \mathbf{u}=f & \text { in } \Omega, \\
\mathbf{u}=-a(\nabla p-\mathbf{b}) & \text { in } \Omega, \\
\mathbf{u} \cdot \nu=g & \text { on } \partial \Omega .
\end{array}
$$

The above system of two first order differential equations is described as a mixed formulation, and it is preferable to a single second order differential equation for $p$ because it allows one to enforce the conservation property for the flux (1.1) locally [16]. An example governed by this system is fluid flow in porous media, where the permeability (divided by fluid viscosity) $a$ can vary by many orders of magnitude over a small spatial displacement, $\mathbf{u}$ is the Darcy velocity, $p$ is the fluid pressure, and $f$ models sources and sinks, i.e., wells, which themselves may be quite small scale features in the problem.

To approximate the velocity $\mathbf{u}$ and pressure $p$ on the coarse mesh $\mathcal{T}_{H}$ requires meeting two competing objectives. First, the approximating spaces must be rich enough to follow the variability in the solution. While a fully fine scale approximating space fulfills this objective, it is not computationally efficient. The second objective is to somehow reduce the problem to the size and complexity of an ordinary coarse scale approximation. The natural approach is to simplify the representation of the solution on the coarse element edges in two dimensions, or faces in three dimensions.

In the variational point of view taken by Arbogast et al., the solution space is decomposed into coarse and fine scale components. This also splits the trial space, and therefore the equations, into coarse and fine scale parts. The fine scale equations are local, and thus solvable, and allow one to compute the fine scale part of the solution from the coarse scale part. The problem then reduces to solving a coarse scale problem for the coarse part of the solution. Any of the usual mixed finite element spaces can be used on the coarse scale. To obtain good approximation on the coarse element edges or faces in this context, it was found that one should use at least second order accurate velocities on the coarse scale.

The multiscale finite element approach of $\mathrm{Hou}, \mathrm{Wu}$, and Cai is based on using the lowest order Raviart-Thomas (RT0) spaces [27] on the coarse scale. One modifies the usual coarse basis to incorporate the microstructure in $a$ by solving the system (1.1)-(1.2) locally. This produces finite elements that vary much like the solution itself. One simply solves a coarse mesh mixed finite element method using these perturbed elements. However, because the RT0 spaces are only first order accurate, they do not give good approximation on the coarse element boundaries. To alleviate this problem, Hou, Wu, and Cai propose an oversampling technique, in which they modify each local basis function by sampling the microstructure over a domain larger than its support. This induces variability in the velocity across coarse element edges or faces and improves the quality of the solution. Several interesting and important advances in the design of the mixed multiscale finite elements have been proposed by Aarnes [1] and Aarnes, Krogstad, and Lie [2].

In this paper, we obtain a connection between the two frameworks. Even though they appear very different, we show that they are in fact equivalent under mild restrictions. We first extend the subgrid upscaling approach to allow oversampling. Then the two frameworks are equivalent provided that one uses the RT0 spaces, and provided that there are no fine scale components in $f$. This last is a subtle point, but important in porous media applications, since wells are so small in two of their three dimensions. The variational framework picks up additional terms related to fine scale 
components of the wells that are overlooked in the multiscale finite element approach, since the latter emphasizes only heterogeneity in $a$ (unless perhaps one supplements the finite element space with special well elements).

We also show that the multiscale error analysis of Chen and Hou [17] extends to the variational multiscale framework. In this analysis, one considers $a(x)$ to be locally periodic of period $\epsilon$; that is, the scale of the heterogeneity is well defined as $\epsilon$. In the case considered in [17], RT0 on simplices, our results are similar and give an $O(\epsilon+H+\sqrt{\epsilon / H})$ error bound, wherein the bounding constant depends on Sobolev norms of the smooth homogenized solution but not on the solution itself. Moreover, the proof is elucidated by the application of variational upscaling ideas and results in improved error estimates with regard to $f$, requiring its $L^{2}$-norm to be bounded rather than its $H^{1}$-norm. When oversampling is not used, we obtain error bounds of $O\left(\epsilon+H^{m}+\sqrt{\epsilon / H}\right)$ for RT0 on nonsimplicial elements $(m=1)$ and the second order accurate $(m \leq 2)$ Brezzi-Douglas-Marini (BDM1) [15] spaces in two dimensions or the Brezzi-Douglas-Durán-Fortin (BDDF1) [14] spaces in three dimensions. Furthermore, when oversampling is not used, we obtain an important superconvergence result for the pressure approximation, showing that it is $O((\epsilon+$ $\left.\left.H+(\epsilon / H)^{1 / d-\eta}\right)\left(\epsilon+H^{m}+\sqrt{\epsilon / H}\right)\right)$, where $\eta>0$ if $d=2$ and $\eta=0$ if $d=3$.

The outline of the paper follows. In section 2 , we apply the construction in [5] to obtain equations upscaled to the coarse level. We show that the upscaling correction terms are antidiffusive and nonlocal in character. We also extend the method to allow oversampling. In section 3 , we extract the multiscale finite elements that are implicit in the construction and show when the method is equivalent to that of Chen and Hou [17]. In section 4, we discuss the fundamental inf-sup lemma regarding solvability and approximability. In the next section, section 5, we state certain homogenization results that we need for section 6 , in which our multiscale convergence result for the velocity is stated and proved. Due to the structure of the inf-sup lemma, the error has two components, the optimal velocity error and an error due to the use of nonconforming spaces. Finally, in section 7 , we treat the pressure error and show that it is superconvergent in the multiscale setting.

We close the introduction by recasting (1.1)-(1.3) in variational form. Let

$$
H(\operatorname{div} ; \Omega)=\left\{\mathbf{v} \in\left(L^{2}(\Omega)\right)^{d}: \nabla \cdot \mathbf{v} \in L^{2}(\Omega)\right\}
$$

with inner product

$$
\left(\mathbf{v}_{1}, \mathbf{v}_{2}\right)_{H(\operatorname{div} ; \Omega)}=\left(\mathbf{v}_{1}, \mathbf{v}_{2}\right)_{\left(L^{2}(\Omega)\right)^{d}}+\left(\nabla \cdot \mathbf{v}_{1}, \nabla \cdot \mathbf{v}_{2}\right)_{L^{2}(\Omega)}
$$

and norm $\|\mathbf{v}\|_{H(\operatorname{div} ; \Omega)}=(\mathbf{v}, \mathbf{v})_{H(\operatorname{div} ; \Omega)}^{1 / 2}$. We set

$$
\mathbf{V}=H_{0}(\operatorname{div} ; \Omega)=\{\mathbf{v} \in H(\operatorname{div} ; \Omega): \mathbf{v} \cdot \nu=0 \text { on } \partial \Omega\}
$$

and $W=L^{2}(\Omega) / \mathbb{R}$ with the $L^{2}(\Omega)$-norm, so that $\nabla \cdot \mathbf{V}=W$. We wish to find $\mathbf{u} \in \mathbf{V}+\mathbf{v}_{g}$ and $p \in W$ such that

$$
\begin{array}{ll}
(\nabla \cdot \mathbf{u}, w)=(f, w) & \forall w \in W \\
(\alpha \mathbf{u}, \mathbf{v})=(p, \nabla \cdot \mathbf{v})+(\mathbf{b}, \mathbf{v}) & \forall \mathbf{v} \in \mathbf{V},
\end{array}
$$

where $\alpha=a^{-1}$ and we denote the $L^{2}(S)$ inner product by $(\cdot, \cdot)_{S}$ for set $S$ and omit $S$ from the notation when it is $\Omega$. We assume that $a \in\left(L^{\infty}(\Omega)\right)^{d \times d}, \mathbf{b} \in\left(L^{2}(\Omega)\right)^{d}$, 
$f \in L^{2}(\Omega)$, and $\mathbf{v}_{g} \in H^{1}(\Omega)$. Provided that we have the compatibility condition

$$
\int_{\Omega} f(x) d x=\int_{\partial \Omega} g(x) d s
$$

it follows from the standard inf-sup theory of saddle point problems $[8,12,11,16,17]$ that (1.4)-(1.5) is indeed uniquely solvable.

2. Approximation by the variational multiscale method. Let $\mathcal{T}_{H}$ be a regular and quasi-uniform partition of $\Omega$ into simplices and/or bricks having maximum diameter $H$, satisfying the condition that the minimum angle of each $E$ is bounded below by some positive constant independent of $H$. Consider the orthogonal direct sum decomposition

$$
W=\bar{W} \oplus W^{\prime},
$$

where the coarse space is

$$
\bar{W}=\left\{\bar{w} \in W: \bar{w} \text { is constant on each } E \in \mathcal{T}_{H}\right\}
$$

and the "subgrid" space is the orthogonal complement

$$
W^{\prime}=\bar{W}^{\perp}=\left\{w^{\prime} \in W: \int_{E} w^{\prime}(x) d x=0 \forall E \in \mathcal{T}_{H}\right\} .
$$

Following [5], we can find a (nonorthogonal) direct sum decomposition of $\mathbf{V}$ into closed subspaces $\mathbf{V}$ and $\mathbf{V}^{\prime}$ such that

$$
\begin{aligned}
\mathbf{V} & =\overline{\mathbf{V}} \oplus \mathbf{V}^{\prime}, \\
\overline{\mathbf{V}} & \subseteq\{\overline{\mathbf{v}} \in \mathbf{V}: \nabla \cdot \overline{\mathbf{v}} \in \bar{W}\}, \\
\mathbf{V}^{\prime} & =\left\{\mathbf{v}^{\prime} \in \mathbf{V}: \nabla \cdot \mathbf{v}^{\prime} \in W^{\prime} \text { and } \mathbf{v}^{\prime} \cdot \nu^{E}=0 \text { on } \partial E \quad \forall E \in \mathcal{T}_{H}\right\} ;
\end{aligned}
$$

moreover, $\nabla \cdot \overline{\mathbf{V}}=\bar{W}$ and $\nabla \cdot \mathbf{V}^{\prime}=W^{\prime}$. Thus we can uniquely decompose the solution $(\mathbf{u}, p) \in\left(\mathbf{V}+\mathbf{v}_{g}\right) \times W$ of $(1.4)-(1.5)$ as

$$
\begin{aligned}
& \mathbf{u}=\overline{\mathbf{u}}+\mathbf{u}^{\prime}+\mathbf{v}_{g}, \\
& p=\bar{p}+p^{\prime},
\end{aligned}
$$

where $\overline{\mathbf{u}} \in \overline{\mathbf{V}}, \mathbf{u}^{\prime} \in \mathbf{V}^{\prime}, \bar{p} \in \bar{W}$, and $p^{\prime} \in W^{\prime}$.

2.1. Subgrid closure operators. By using the above decompositions and restricting the test functions in (1.4)-(1.5) to $\left(\mathbf{v}^{\prime}, w^{\prime}\right) \in \mathbf{V}^{\prime} \times W^{\prime}$, we obtain the subgrid equation

$$
\begin{array}{ll}
\left(\nabla \cdot \mathbf{u}^{\prime}, w^{\prime}\right)=\left(f-\nabla \cdot \mathbf{v}_{g}, w^{\prime}\right) & \forall w^{\prime} \in W^{\prime}, \\
\left(\alpha\left(\overline{\mathbf{u}}+\mathbf{u}^{\prime}\right), \mathbf{v}^{\prime}\right)=\left(p^{\prime}, \nabla \cdot \mathbf{v}^{\prime}\right)+\left(\mathbf{b}-\alpha \mathbf{v}_{g}, \mathbf{v}^{\prime}\right) & \forall \mathbf{v}^{\prime} \in \mathbf{V}^{\prime},
\end{array}
$$

where certain terms have vanished due to the orthogonality of $\bar{W}$ and $W^{\prime}$ and the property that $\nabla \cdot \overline{\mathbf{V}}=\bar{W}$. Note that for our problem, $\bar{p}$ does not appear in the above equation (see [5] for handling the general case).

We now define the subgrid closure operators mapping each $\overline{\mathbf{u}} \in \overline{\mathbf{V}}$ to some $\mathbf{u}^{\prime} \in \mathbf{V}^{\prime}$ and $p^{\prime} \in W^{\prime}$. Each is an affine operator consisting of a linear and a constant part depending on $\overline{\mathbf{u}}$, the coarse part of $\mathbf{u}$, so we write

$$
\begin{aligned}
\mathbf{u}^{\prime} & =\mathbf{u}^{\prime}(\overline{\mathbf{u}})=\hat{\mathbf{u}}^{\prime}(\overline{\mathbf{u}})+\tilde{\mathbf{u}}^{\prime}, \\
p^{\prime} & =p^{\prime}(\overline{\mathbf{u}})=\hat{p}^{\prime}(\overline{\mathbf{u}})+\tilde{p}^{\prime}
\end{aligned}
$$


More generally, for each $\mathbf{v} \in H(\operatorname{div} ; \Omega)$, by $(2.3)-(2.4),\left(\hat{\mathbf{u}}^{\prime}(\mathbf{v}), \hat{p}^{\prime}(\mathbf{v})\right) \in \mathbf{V}^{\prime} \times W^{\prime}$ is defined by

$$
\begin{array}{ll}
\left(\nabla \cdot \hat{\mathbf{u}}^{\prime}(\mathbf{v}), w^{\prime}\right)=0 & \forall w^{\prime} \in W^{\prime}, \\
\left(\alpha\left(\mathbf{v}+\hat{\mathbf{u}}^{\prime}(\mathbf{v})\right), \mathbf{v}^{\prime}\right)=\left(\hat{p}^{\prime}(\mathbf{v}), \nabla \cdot \mathbf{v}^{\prime}\right) & \forall \mathbf{v}^{\prime} \in \mathbf{V}^{\prime},
\end{array}
$$

and $\left(\tilde{\mathbf{u}}^{\prime}, \tilde{p}^{\prime}\right) \in \mathbf{V}^{\prime} \times W^{\prime}$ is defined by

$$
\begin{array}{ll}
\left(\nabla \cdot \tilde{\mathbf{u}}^{\prime}, w^{\prime}\right)=\left(f-\nabla \cdot \mathbf{v}_{g}, w^{\prime}\right) & \forall w^{\prime} \in W^{\prime}, \\
\left(\alpha \tilde{\mathbf{u}}^{\prime}, \mathbf{v}^{\prime}\right)=\left(\tilde{p}^{\prime}, \nabla \cdot \mathbf{v}^{\prime}\right)+\left(\mathbf{b}-\alpha \mathbf{v}_{g}, \mathbf{v}^{\prime}\right) & \forall \mathbf{v}^{\prime} \in \mathbf{V}^{\prime} .
\end{array}
$$

These equations are well-posed on each $E \in \mathcal{T}_{H}[5]$.

For future reference, we note that on each $E \in \mathcal{T}_{H}$,

$$
\begin{aligned}
& -a \nabla \hat{p}^{\prime}(\mathbf{v})=\mathbf{v}+\hat{\mathbf{u}}^{\prime}(\mathbf{v}), \\
& -a \nabla \tilde{p}^{\prime}=\tilde{\mathbf{u}}^{\prime}-a \mathbf{b}+\mathbf{v}_{g},
\end{aligned}
$$

because $\left.\mathbf{V}^{\prime}\right|_{E}=H_{0}($ div; $E)$ is the full space. Moreover, $\left.W^{\prime}\right|_{E}=L^{2}(E) / \mathbb{R}$, so

$$
\nabla \cdot \hat{\mathbf{u}}^{\prime}(\mathbf{v})=0 .
$$

2.2. The upscaled equation. We now define a vector space $\hat{\mathbf{V}} \subseteq \mathbf{V}$ by

$$
\hat{\mathbf{V}}=\left\{\hat{\mathbf{v}} \in \mathbf{V}: \hat{\mathbf{v}}=\overline{\mathbf{v}}+\hat{\mathbf{u}}^{\prime}(\overline{\mathbf{v}}) \text { for some } \overline{\mathbf{v}} \in \overline{\mathbf{V}}\right\},
$$

restrict the test functions in (1.4)-(1.5) to be in $\hat{\mathbf{V}} \times \bar{W}$, use the various decompositions, and introduce the notation

$$
\hat{f}=f-\nabla \cdot \mathbf{v}_{g} \quad \text { and } \quad \hat{\mathbf{b}}=\mathbf{b}-\alpha\left(\mathbf{v}_{g}+\tilde{\mathbf{u}}^{\prime}\right) .
$$

Thus we rewrite (1.4)-(1.5) in upscaled form as the problem of finding $(\hat{\mathbf{u}}, \bar{p}) \in \hat{\mathbf{V}} \times \bar{W}$ such that

$$
\begin{array}{ll}
(\nabla \cdot \hat{\mathbf{u}}, \bar{w})=(\hat{f}, \bar{w}) & \forall \bar{w} \in \bar{W}, \\
(\alpha \hat{\mathbf{u}}, \hat{\mathbf{v}})=(\bar{p}, \nabla \cdot \hat{\mathbf{v}})+(\hat{\mathbf{b}}, \hat{\mathbf{v}}) & \forall \hat{\mathbf{v}} \in \hat{\mathbf{V}},
\end{array}
$$

where now

$$
\mathbf{u}=\overline{\mathbf{u}}+\hat{\mathbf{u}}^{\prime}(\overline{\mathbf{u}})+\tilde{\mathbf{u}}^{\prime}+\mathbf{v}_{g}=\hat{\mathbf{u}}+\tilde{\mathbf{u}}^{\prime}+\mathbf{v}_{g} .
$$

By [5, Theorem 4.6], the above problem has a unique solution.

2.3. Character of the upscaled operator. With $\mathbf{v}^{\prime}=\hat{\mathbf{u}}^{\prime}(\overline{\mathbf{u}})$ in $(2.8)$, we note that

$$
\left(\alpha \hat{\mathbf{u}}^{\prime}(\overline{\mathbf{u}}), \overline{\mathbf{v}}\right)=-\left(\alpha \hat{\mathbf{u}}^{\prime}(\overline{\mathbf{u}}), \hat{\mathbf{u}}^{\prime}(\overline{\mathbf{v}})\right),
$$

so (1.5) with $\mathbf{v}=\overline{\mathbf{v}} \in \overline{\mathbf{V}}$ enables us to rewrite the upscaled equation (2.15) as

$$
(\alpha \overline{\mathbf{u}}, \overline{\mathbf{v}})-\left(\alpha \hat{\mathbf{u}}^{\prime}(\overline{\mathbf{u}}), \hat{\mathbf{u}}^{\prime}(\overline{\mathbf{v}})\right)=(\bar{p}, \nabla \cdot \overline{\mathbf{v}})+(\hat{\mathbf{b}}, \overline{\mathbf{v}}) \quad \forall \overline{\mathbf{v}} \in \overline{\mathbf{V}} .
$$

Thus the second term on the left-hand side, the primary subscale correction, is purely antidiffusive on the coarse scale, as we should expect. Moreover, there is an affine correction term related to subscales of $\mathbf{b}, f$, and $\mathbf{v}_{g}$ through $\tilde{\mathbf{u}}^{\prime}$. 
Next let $G_{x}(y)$ be the Green's function on a coarse element $E$, defined by

$$
\begin{array}{ll}
-\nabla \cdot a \nabla G_{x}=\delta_{x}-1 /|E| & \text { in } E, \\
-a \nabla G_{x} \cdot \nu^{E}=0 & \text { on } \partial E,
\end{array}
$$

where $\delta_{x}$ is the Dirac mass at $x \in E$ and the average of $G_{x}$ vanishes, and where vertical bars around a set in $\mathbb{R}^{d}$ denotes $d$-dimensional or $(d-1)$-dimensional Lebesgue measure, as appropriate. Then on $E$,

$$
\begin{aligned}
p(x) & =\left(-\nabla \cdot a \nabla G_{x}+1 /|E|, p\right)_{E} \\
& =\left(a \nabla G_{x}, \nabla p\right)_{E}+\bar{p} \\
& =-\left(\nabla G_{x}, \mathbf{u}-a \mathbf{b}\right)_{E}+\bar{p} \\
& =-\left(\nabla G_{x}, \overline{\mathbf{u}}-a \mathbf{b}\right)_{E}-\left(\nabla G_{x}, \mathbf{u}^{\prime}+\mathbf{v}_{g}\right)_{E}+\bar{p} \\
& =-\left(\nabla G_{x}, \overline{\mathbf{u}}-a \mathbf{b}\right)_{E}+\left(G_{x}, \nabla \cdot\left(\mathbf{u}^{\prime}+\mathbf{v}_{g}\right)\right)_{E}-\left(G_{x}, \mathbf{v}_{g} \cdot \nu^{E}\right)_{\partial E}+\bar{p} \\
& =-\left(\nabla G_{x}, \overline{\mathbf{u}}-a \mathbf{b}\right)_{E}+\left(G_{x}, f^{\prime}\right)_{E}-\left(G_{x}, \mathbf{v}_{g} \cdot \nu^{E}\right)_{\partial E}+\bar{p},
\end{aligned}
$$

where $f^{\prime}$ is defined by the decomposition $f=\bar{f}+f^{\prime} \in \bar{W} \oplus W^{\prime}$ and we use that $G_{x} \in W^{\prime}$ to replace $\left(G_{x}, \nabla \cdot\left(\mathbf{u}^{\prime}+\mathbf{v}_{g}\right)\right)_{E}$ by $\left(G_{x}, \nabla \cdot \mathbf{u}\right)_{E}=\left(G_{x}, f^{\prime}\right)$. Now

$$
\alpha(x) \mathbf{u}(x)-\mathbf{b}=-\nabla p=\left(\nabla_{x} \nabla_{y} G_{x}, \overline{\mathbf{u}}-a \mathbf{b}\right)_{E}-\left(\nabla_{x} G_{x}, f^{\prime}\right)_{E}+\left(\nabla_{x} G_{x}, \mathbf{v}_{g} \cdot \nu^{E}\right)_{\partial E},
$$

so the diffusive and $\mathbf{b}$ terms of (1.5), tested on the coarse scale, are

$$
\begin{aligned}
(\alpha \mathbf{u}-\mathbf{b}, \overline{\mathbf{v}})_{E}= & \int_{E} \int_{E} \overline{\mathbf{u}}(y) \cdot \nabla_{x} \nabla_{y} G_{x}(x, y) \cdot \overline{\mathbf{v}}(x) d y d x \\
& -\int_{E} \int_{E} \mathbf{b}(y) \cdot a(y) \nabla_{x} \nabla_{y} G_{x}(x, y) \cdot \overline{\mathbf{v}}(x) d y d x \\
& -\int_{E} \int_{E} f^{\prime}(y) \nabla_{x} G_{x}(x, y) \cdot \overline{\mathbf{v}}(x) d y d x \\
& +\int_{E} \int_{\partial E} \mathbf{v}_{g} \cdot \nu^{E}(y) \nabla_{x} G_{x}(x, y) \cdot \overline{\mathbf{v}}(x) d s(y) d x,
\end{aligned}
$$

so the upscaled inverse permeability tensor is a nonlocal operator (confined to $E$ ) related to $a(y) \nabla_{x} \nabla_{y} G_{x}(y)$.

2.4. Oversampling. For each element $E \in \mathcal{T}_{H}$, choose some larger set $E_{*} \supseteq E$ such that $E_{*} \subseteq \Omega, E_{*}$ is the same shape as $E$ (i.e., a simplex or brick, again such that the minimum angle is bounded below by some positive constant independent of $H$ and $E$ ), and, for some $C>0$ independent of $H$ and $E, \operatorname{diam}\left(E_{*}\right) \leq C \operatorname{diam}(E)$. Locally on each $E_{*}$, recalling the definition of $W^{\prime}$ and properties of $\mathbf{V}^{\prime}$, we define function spaces $W_{*}^{\prime}\left(E_{*}\right)=L^{2}\left(E_{*}\right) / \mathbb{R}$ and

$$
\mathbf{V}_{*}^{\prime}\left(E_{*}\right)=\left\{\mathbf{v}_{*}^{\prime} \in \mathbf{V}: \nabla \cdot \mathbf{v}_{*}^{\prime} \in W_{*}^{\prime}\left(E_{*}\right) \text { and } \mathbf{v}_{*}^{\prime} \cdot \nu^{E_{*}}=0 \text { on } \partial E_{*}\right\} .
$$

By analogy to (2.7)-(2.8), we now define the linear part of the oversampled subgrid closure operators mapping any $\mathbf{v} \in \mathbf{V}$ to some $\left(\hat{\mathbf{u}}_{*}^{\prime}(\mathbf{v}), \hat{p}_{*}^{\prime}(\mathbf{v})\right) \in \mathbf{V}_{*}^{\prime}\left(E_{*}\right) \times W_{*}^{\prime}\left(E_{*}\right)$ defined by

$$
\begin{array}{ll}
\left(\nabla \cdot \hat{\mathbf{u}}_{*}^{\prime}(\mathbf{v}), w_{*}^{\prime}\right)_{E_{*}}=0 & \forall w_{*}^{\prime} \in W_{*}^{\prime}\left(E_{*}\right), \\
\left(\alpha\left(\mathbf{v}+\hat{\mathbf{u}}_{*}^{\prime}(\mathbf{v})\right), \mathbf{v}_{*}^{\prime}\right)_{E_{*}}=\left(\hat{p}_{*}^{\prime}(\mathbf{v}), \nabla \cdot \mathbf{v}_{*}^{\prime}\right)_{E_{*}} & \forall \mathbf{v}_{*}^{\prime} \in \mathbf{V}_{*}^{\prime}\left(E_{*}\right) .
\end{array}
$$


Note that if $E_{*}=E$, then the operators $\hat{\mathbf{u}}_{*}^{\prime}(\cdot)$ and $\hat{\mathbf{u}}^{\prime}(\cdot)$ coincide. We also define the oversampled constant parts of the subgrid closure operators corresponding to (2.9)$(2.10)$ as $\left(\tilde{\mathbf{u}}_{*}^{\prime}, \tilde{p}_{*}^{\prime}\right) \in \mathbf{V}_{*}^{\prime}\left(E_{*}\right) \times W_{*}^{\prime}\left(E_{*}\right)$ defined by

$$
\begin{array}{ll}
\left(\nabla \cdot \tilde{\mathbf{u}}_{*}^{\prime}, w_{*}^{\prime}\right)_{E_{*}}=\left(f-\nabla \cdot \mathbf{v}_{g}, w_{*}^{\prime}\right)_{E_{*}} & \forall w_{*}^{\prime} \in W_{*}^{\prime}\left(E_{*}\right), \\
\left(\alpha \tilde{\mathbf{u}}_{*}^{\prime}, \mathbf{v}_{*}^{\prime}\right)_{E_{*}}=\left(\tilde{p}_{*}^{\prime}, \nabla \cdot \mathbf{v}_{*}^{\prime}\right)_{E_{*}}+\left(\mathbf{b}-\alpha \mathbf{v}_{g}, \mathbf{v}_{*}^{\prime}\right)_{E_{*}} & \forall \mathbf{v}_{*}^{\prime} \in \mathbf{V}_{*}^{\prime}\left(E_{*}\right) .
\end{array}
$$

Usually we consider these quantities only locally on $E$, so we need not concern ourselves with the overlap of the $E_{*}$ 's. Also note that $\nabla \cdot \hat{\mathbf{u}}_{*}^{\prime}(\mathbf{v})=0$ for all $\mathbf{v} \in \mathbf{V}$.

2.5. Discretization. In practice, we must approximate the solution to the subgrid problems $(2.20)-(2.21)$ and $(2.22)-(2.23)$. Since these problems are small (i.e., localized to $E_{*}$ ), we assume that we can fully resolve the fine scales in these problems on a fine subgrid mesh and thereby obtain a sufficiently accurate approximation (see also [5]). Thus, we will discuss only approximation of the coarse space in this paper, and we assume that the subgrid is solved exactly.

Let $\overline{\mathbf{V}}_{H} \times \bar{W}_{H} \subseteq \mathbf{V} \times W$ be the lowest order Raviart-Thomas (RT0) [27] space or the lowest order Brezzi-Douglas-Marini (BDM1) [15] space in two dimensions or the Brezzi-Douglas-Durán-Fortin (BDDF1) [14] space in three dimensions. In each case, the pressure approximation space is the space of piecewise constants, so we have $\bar{W}_{H}=\bar{W}$. Let $\mathcal{E}_{E}$ be the analytic extension operator from $E$ to $E_{*}$, and define the function space

$$
\hat{\mathbf{v}}_{H, *}=\left\{\hat{\mathbf{v}}_{H, *}: \hat{\mathbf{v}}_{H, *}=\overline{\mathbf{v}}_{H}+\left.\sum_{E \in \mathcal{T}_{H}} \hat{\mathbf{u}}_{*}^{\prime}\left(\mathcal{E}_{E} \overline{\mathbf{v}}_{H}\right)\right|_{E} \text { for some } \overline{\mathbf{v}}_{H} \in \overline{\mathbf{V}}_{H}\right\},
$$

wherein, technically, $\mathcal{E}_{E} \overline{\mathbf{v}}_{H}=\mathcal{E}_{E}\left(\left.\overline{\mathbf{v}}_{H}\right|_{E}\right)$. Now $\hat{\mathbf{V}}_{H, *} \subseteq X$, where

$$
X=\bigoplus_{E \in \mathcal{T}_{H}} H(\operatorname{div} ; E)
$$

is a Banach space with the norm $\|\mathbf{v}\|_{X}=\left(\sum_{E \in \mathcal{T}_{H}}\|\mathbf{v}\|_{H(\text { div;E) }}^{2}\right)^{1 / 2}$. Clearly $\mathbf{V}=$ $H_{0}(\operatorname{div} ; \Omega) \subseteq X$, but if $E_{*} \neq E$ for any $E \in \mathcal{T}_{H}$, then $\hat{\mathbf{V}}_{H, *} \nsubseteq \mathbb{V}$ and we have a nonconforming finite element space.

We approximate (2.14)-(2.15) by the problem of finding $\left(\hat{\mathbf{u}}_{H}, \bar{p}_{H}\right) \in \hat{\mathbf{V}}_{H, *} \times \bar{W}_{H}$ such that

$$
\begin{array}{ll}
\sum_{E \in \mathcal{T}_{H}}\left(\nabla \cdot \hat{\mathbf{u}}_{H}, \bar{w}_{H}\right)_{E}=\left(\hat{f}, \bar{w}_{H}\right) & \forall \bar{w}_{H} \in \bar{W}_{H}, \\
\left(\alpha \hat{\mathbf{u}}_{H}, \hat{\mathbf{v}}_{H}\right)=\sum_{E \in \mathcal{T}_{H}}\left(\bar{p}_{H}, \nabla \cdot \hat{\mathbf{v}}_{H}\right)_{E}+\left(\hat{\mathbf{b}}_{*}, \hat{\mathbf{v}}_{H}\right) & \forall \hat{\mathbf{v}}_{H} \in \hat{\mathbf{V}}_{H, *},
\end{array}
$$

where

$$
\hat{\mathbf{b}}_{*}=\mathbf{b}-\alpha\left(\mathbf{v}_{g}+\left.\sum_{E \in \mathcal{T}_{H}} \tilde{\mathbf{u}}_{*}^{\prime}\right|_{E}\right)
$$

Define the affine space

$$
\mathbf{V}_{H, *}=\hat{\mathbf{V}}_{H, *}+\left.\sum_{E \in \mathcal{T}_{H}} \tilde{\mathbf{u}}_{*}^{\prime}\right|_{E}+\mathbf{v}_{g}
$$


and the discrete oversampled approximation

$$
\begin{aligned}
& \mathbf{u} \approx \mathbf{u}_{H}=\hat{\mathbf{u}}_{H}+\left.\sum_{E \in \mathcal{T}_{H}} \tilde{\mathbf{u}}_{*}^{\prime}\right|_{E}+\mathbf{v}_{g} \in \mathbf{V}_{H, *}, \\
& p \approx p_{H}=\bar{p}_{H}+\left.\sum_{E \in \mathcal{T}_{H}}\left(\hat{p}_{*}^{\prime}\left(\mathcal{E}_{E} \overline{\mathbf{u}}_{H}\right)+\tilde{p}_{*}^{\prime}\right)\right|_{E} \in W .
\end{aligned}
$$

The full approximation satisfies

$$
\begin{array}{ll}
\sum_{E \in \mathcal{T}_{H}}\left(\nabla \cdot \mathbf{u}_{H}, w\right)_{E}=(f, w) & \forall w \in W \\
\left(\alpha \mathbf{u}_{H}, \hat{\mathbf{v}}_{H}\right)=\sum_{E \in \mathcal{T}_{H}}\left(\bar{p}_{H}, \nabla \cdot \hat{\mathbf{v}}_{H}\right)_{E}+\left(\mathbf{b}, \hat{\mathbf{v}}_{H}\right) & \forall \hat{\mathbf{v}}_{H} \in \hat{\mathbf{V}}_{H, *},
\end{array}
$$

which corresponds to the original system (1.4)-(1.5). The systems (2.26)-(2.27) and (2.31)-(2.32) are equivalent; the former is suitable for computation and the latter for analysis. In section 4 , it will be shown using the abstract inf-sup lemma $[8,12,11$, $16,17]$ that this problem has a unique solution.

If oversampling is not used, this is the same discrete approximation considered in [5], except that there the subgrid operators are also approximated on a finer mesh than $\mathcal{T}_{H}$. Since our concern in this paper is to relate $\epsilon$, the scale of the heterogeneity, to $H$, the size of the coarse mesh, we have assumed that the subgrid operators are fully resolved (as was done in [17]).

3. Partial equivalence with the multiscale finite element method. In [17], Chen and Hou give a mixed finite element method for the equations making use of their multiscale finite element basis functions. As we show in this section, their method is fundamentally equivalent to that described in this paper in the case where $\overline{\mathbf{V}}_{H}$ is the vector variable part of the RT0 space and $\tilde{\mathbf{u}}^{\prime}$ and $\tilde{p}^{\prime}$ vanish. Note that from (2.9)-(2.10), $\tilde{\mathbf{u}}^{\prime}$ and $\tilde{p}^{\prime}$ vanish exactly when $\left(f-\nabla \cdot \mathbf{v}_{g}, w^{\prime}\right)=0$ for all $w^{\prime} \in W^{\prime}$ and $\left(\mathbf{b}-\alpha \mathbf{v}_{g}, \mathbf{v}^{\prime}\right)=0$ for all $\mathbf{v}^{\prime} \in \mathbf{V}^{\prime}$; that is, the subscales of $f-\nabla \cdot \mathbf{v}_{g}$ and $\mathbf{b}-\alpha \mathbf{v}_{g}$ vanish.

Let $E \in \mathcal{T}_{H}$ be given, and let $e_{i}^{E}$ represent the $i$ th edge in two dimensions or face in three dimensions of $E$. We begin by recalling a standard basis $\left\{R_{i}^{E}\right\}$ for $\operatorname{RT}_{0}(E)$, the vector part of the RT0 space [27] on $E \in \mathcal{T}_{H}$, which satisfies

$$
\begin{array}{ll}
\nabla \cdot R_{i}^{E}=1 /|E| & \text { in } E, \\
R_{i}^{E}=-\nabla \omega_{i}^{E} & \text { in } E, \\
R_{i}^{E} \cdot \nu^{E}=\left\{\begin{aligned}
1 /\left|e_{i}^{E}\right| & \text { on } e_{i}^{E}, \\
0 & \text { on } e_{j}^{E}, j \neq i .
\end{aligned}\right.
\end{array}
$$

That is, $R_{i}^{E}$ is linear, has a constant divergence, and has constant fluxes over the edges or faces of $E$.

Chen and Hou [17] construct the multiscale finite element space in the following way. Let $\left\{R_{i}^{E_{*}}\right\}$ be the basis of $\operatorname{RT}_{0}\left(E_{*}\right)$, the vector part of the RT0 space on $E_{*}$, which satisfies

$$
R_{i}^{E_{*}} \cdot \nu^{E^{*}}= \begin{cases}1 /\left|e_{i}^{E_{*}}\right| & \text { on } e_{i}^{E_{*}}, \\ 0 & \text { on } e_{j}^{E_{*}}, j \neq i\end{cases}
$$


where $e_{i}^{E_{*}}$ represents an edge or face of $E_{*}$. Since the RT0 basis functions on $E_{*}$ also span $\mathrm{RT}_{0}(E)$, there must exist, for each $i$ and $j$, constants $c_{i j}^{E}$ such that

$$
R_{i}^{E}=\left.\sum_{j} c_{i j}^{E} R_{j}^{E_{*}}\right|_{E}
$$

(If $E_{*}=E$, we simply have $c_{i j}^{E}=\delta_{i j}$, where $\delta_{i j}$ is the Kronecker delta.) Now for each $j$, let $w_{j}^{E_{*}}$ be the unique solution in $L^{2}\left(E_{*}\right) / \mathbb{R}=W_{*}^{\prime}\left(E_{*}\right)$ of the Neumann problem

$$
\int_{E_{*}} a \nabla w_{j}^{E_{*}} \cdot \nabla \varphi d x=\frac{1}{\left|E_{*}\right|} \int_{E_{*}} \varphi d x-\frac{1}{\left|e_{j}^{E_{*}}\right|} \int_{e_{j}^{E_{*}}} \varphi d s \quad \forall \varphi \in H^{1}\left(E_{*}\right),
$$

which is equivalent to

$$
\begin{array}{ll}
\nabla \cdot \hat{\psi}_{H, i}=\nabla \cdot R_{i}^{E_{*}} & \text { in } E_{*}, \\
\hat{\psi}_{H, i}=-a \nabla w_{i}^{E_{*}} & \text { in } E_{*}, \\
\hat{\psi}_{H, i} \cdot \nu^{E_{*}}=R_{i}^{E_{*}} \cdot \nu^{E_{*}} & \text { on } \partial E_{*} .
\end{array}
$$

For each $i$, set

$$
\tilde{w}_{i}^{E_{*}}=\sum_{j} c_{i j}^{E} w_{j}^{E_{*}}
$$

Now let

$$
\operatorname{MS}_{*}(E)=\operatorname{span}\left\{-\left.a \nabla \tilde{w}_{i}^{E_{*}}\right|_{E}\right\}
$$

and $\tilde{X}_{H, *}=\left\{\mathbf{v} \in X:\left.\mathbf{v}\right|_{E} \in \operatorname{MS}_{*}(E)\right.$ for all $\left.E \in \mathcal{T}_{H}\right\}$. Define

$$
\Pi_{H}: \tilde{X}_{H, *} \rightarrow \bigoplus_{E \in \mathcal{T}_{H}} \mathrm{RT}_{0}(E)
$$

to be the natural projection defined locally for $\left.\mathbf{v}\right|_{E}=-\sum_{i} b_{i} a \nabla \tilde{w}_{i}^{E}$ by $\left.\Pi_{H}(\mathbf{v})\right|_{E}=$ $\sum_{i} b_{i} R_{i}^{E}$. The oversampled multiscale finite element space $\hat{X}_{H, *} \subseteq X$ is then defined by

$$
\hat{X}_{H, *}=\left\{\mathbf{v} \in \tilde{X}_{H, *}: \Pi_{H} \mathbf{v} \in \overline{\mathbf{V}}_{H}\right\},
$$

wherein $\overline{\mathbf{V}}_{H}$ is $\mathrm{RT}_{0}$ in this section. Note that again the subgrid problems have been assumed to be solved exactly, since the fine scales can be fully resolved.

To see the equivalence with the construction in this paper, first note that the problems (3.1), i.e., (3.2)-(3.4), and (2.20)-(2.21) are closely related, so that

$$
-a \nabla w_{j}^{E_{*}}=R_{j}^{E_{*}}+\hat{\mathbf{u}}_{*}^{\prime}\left(R_{j}^{E_{*}}\right)=-a \nabla \hat{p}_{*}^{\prime}\left(R_{j}^{E_{*}}\right) ;
$$

that is, $w_{j}^{E_{*}}=\hat{p}_{*}^{\prime}\left(R_{j}^{E_{*}}\right)$. Now clearly $\mathcal{E}_{E} R_{i}^{E}=\sum_{j} c_{i j}^{E} R_{j}^{E_{*}}$, so

$$
\mathcal{E}_{E} R_{i}^{E}+\hat{\mathbf{u}}_{*}^{\prime}\left(\mathcal{E}_{E} R_{i}^{E}\right)=-a \nabla \hat{p}_{*}^{\prime}\left(\mathcal{E}_{E} R_{i}^{E}\right)=-\sum_{j} c_{i j}^{E} a \nabla \tilde{w}_{j}^{E_{*}} .
$$

Since the matrix $c_{i j}^{E}$ is invertible,

$$
\operatorname{MS}_{*}(E)=\operatorname{span}\left\{R_{i}^{E}+\left.\hat{\mathbf{u}}_{*}^{\prime}\left(\mathcal{E}_{E} R_{i}^{E}\right)\right|_{E}\right\} .
$$


Now if $\mathbf{v} \in \tilde{X}_{H, *}$, then $\left.\mathbf{v}\right|_{E}=\sum_{i} b_{i}\left(R_{i}^{E}+\left.\hat{\mathbf{u}}_{*}^{\prime}\left(\mathcal{E}_{E} R_{i}^{E}\right)\right|_{E}\right)$, and so $\left.\Pi_{H} \mathbf{v}\right|_{E}=\sum_{i} b_{i} R_{i}^{E}$. The condition that $\Pi_{H} \mathbf{v} \in \overline{\mathbf{V}}_{H}$ merely says that the local RT0 basis functions fit together globally in $H(\operatorname{div} ; \Omega)$. Thus $\hat{X}_{H, *}$ is the span of $\overline{\mathbf{v}}_{H}+\left.\sum_{E} \hat{\mathbf{u}}_{*}^{\prime}\left(\mathcal{E}_{E} \overline{\mathbf{v}}_{H}\right)\right|_{E}$ for $\overline{\mathbf{v}}_{H} \in \overline{\mathbf{V}}_{H}$; that is,

$$
\hat{X}_{H, *}=\hat{\mathbf{V}}_{H, *},
$$

and our construction agrees with that in [17], up to the treatment of $\tilde{\mathbf{u}}^{\prime}$ and $\tilde{p}^{\prime}$. Moreover, the mixed multiscale finite element method obtains only $\bar{p}_{H} \in \bar{W}_{H}$ (not $\left.p_{H}\right)$ from $(2.30)$.

4. Analysis of the saddle point variational problem. In this paper, we use the notation $\|\cdot\|_{j, S}$ for the norm of the Sobolev space $H^{j}(S)$, and $\|\cdot\|_{j, p, S}$ for the norm of the Sobolev space $W^{j, p}(S)$ when $p \neq 2$. We proceed through a series of lemmas.

Lemma 4.1. There exists $C>0$, independent of $\epsilon$ and $H$, such that for each $E \in \mathcal{T}_{H}$ and $\mathbf{v} \in \mathbf{V}$,

$$
\left\|\hat{\mathbf{u}}_{*}^{\prime}(\mathbf{v})\right\|_{0, E_{*}}+\left\|\nabla \hat{p}_{*}^{\prime}(\mathbf{v})\right\|_{0, E_{*}} \leq C\|\mathbf{v}\|_{0, E_{*}}
$$

Moreover, if $\mathbf{v} \in \mathbf{V}_{*}^{\prime}\left(E_{*}\right)$ has vanishing divergence, then $\hat{\mathbf{u}}_{*}^{\prime}(\mathbf{v})=-\mathbf{v}$ and $\hat{p}_{*}^{\prime}(\mathbf{v})=0$.

Proof. This is the standard energy estimate for the differential system (2.20)(2.21), and the bound depends only on the ellipticity and continuity constants for $a$ and so is independent of $\epsilon$ and $H$. The final remark is obvious from the definition of the operator.

LEMma 4.2. There exists $C>0$, independent of $\epsilon$ and $H$, such that for any $\hat{\mathbf{v}}_{H} \in \hat{\mathbf{V}}_{H, *}$, if $\overline{\mathbf{v}}_{H} \in \overline{\mathbf{V}}_{H}$ is any element corresponding to $\hat{\mathbf{v}}_{H}$ in the sense that

$$
\hat{\mathbf{v}}_{H}=\overline{\mathbf{v}}_{H}+\left.\sum_{E \in \mathcal{T}_{H}} \hat{\mathbf{u}}_{*}^{\prime}\left(\mathcal{E}_{E} \overline{\mathbf{v}}_{H}\right)\right|_{E}
$$

then on any $E,\left.\nabla \cdot \hat{\mathbf{v}}_{H}\right|_{E}=\left.\nabla \cdot \overline{\mathbf{v}}_{H}\right|_{E}$ and

$$
\left\|\hat{\mathbf{v}}_{H}\right\|_{H(\operatorname{div} ; E)} \leq C\left\|\overline{\mathbf{v}}_{H}\right\|_{H(\operatorname{div} ; E)} .
$$

Proof. By the definition (2.24), each $\hat{\mathbf{v}}_{H} \in \hat{\mathbf{V}}_{H, *}$ has at least one $\overline{\mathbf{v}}_{H}$ satisfying (4.1). Since the operator norm of $\mathcal{E}_{E}$ (as applied to low order polynomials and with respect to the $L^{2}(E)$ - and $L^{2}\left(E_{*}\right)$-norms) is bounded uniformly in $E$ and $H$ under our assumptions on the shape regularity of $E$ and $E_{*}$, we have

$$
\left\|\mathcal{E}_{E} \overline{\mathbf{v}}_{H}+\hat{\mathbf{u}}_{*}^{\prime}\left(\mathcal{E}_{E} \overline{\mathbf{v}}_{H}\right)\right\|_{0, E_{*}} \leq C\left\|\mathcal{E}_{E} \overline{\mathbf{v}}_{H}\right\|_{0, E_{*}} \leq C\left\|\overline{\mathbf{v}}_{H}\right\|_{0, E}
$$

Note that $\mathcal{E}_{E} \overline{\mathbf{v}}_{H}+\hat{\mathbf{u}}_{*}^{\prime}\left(\mathcal{E}_{E} \overline{\mathbf{v}}_{H}\right)$ agrees with $\hat{\mathbf{v}}_{H}$ on $E$, so we have

$$
\left\|\hat{\mathbf{v}}_{H}\right\|_{0, E} \leq C\left\|\overline{\mathbf{v}}_{H}\right\|_{0, E} .
$$

The above inequality holds for the $H(\operatorname{div} ; E)$-norm as well, because $\nabla \cdot \hat{\mathbf{u}}_{*}^{\prime}(\mathbf{v})$ is identically zero for all $\mathbf{v} \in \mathbf{V}$, which implies $\nabla \cdot \hat{\mathbf{v}}_{H}=\nabla \cdot \overline{\mathbf{v}}_{H}$. 
Lemma 4.3. There exists a constant $\beta>0$, independent of $\epsilon$ and $H$, such that for any $\bar{q}_{H} \in \bar{W}_{H}$, the following inf-sup condition holds:

$$
\sup _{0 \neq \hat{\mathbf{v}}_{H} \in \hat{\mathbf{v}}_{H, *}} \frac{\sum_{E \in \mathcal{T}_{H}}\left(\bar{q}_{H}, \nabla \cdot \hat{\mathbf{v}}_{H}\right)_{E}}{\left\|\hat{\mathbf{v}}_{H}\right\|_{X}} \geq \beta\left\|\bar{q}_{H}\right\|_{0, \Omega} .
$$

Proof. It is known that the inf-sup condition holds for all the usual mixed finite element spaces, such as $\bar{W}_{H} \times \overline{\mathbf{V}}_{H}$. Because $\nabla \cdot \hat{\mathbf{u}}_{*}^{\prime}(\cdot)=0$, and by Lemma 4.2 , we have

$$
\sup _{0 \neq \hat{\mathbf{v}}_{H} \in \hat{\mathbf{v}}_{H, *}} \frac{\sum_{E \in \mathcal{T}_{H}}\left(\bar{q}_{H}, \nabla \cdot \hat{\mathbf{v}}_{H}\right)_{E}}{\left\|\hat{\mathbf{v}}_{H}\right\|_{X}} \geq C \sup _{0 \neq \overline{\mathbf{v}}_{H} \in \overline{\mathbf{v}}_{H}} \frac{\left(\bar{q}_{H}, \nabla \cdot \overline{\mathbf{v}}_{H}\right)}{\left\|\overline{\mathbf{v}}_{H}\right\|_{H(\operatorname{div} ; \Omega)}} \geq C \bar{\beta}\left\|\bar{q}_{H}\right\|_{0, \Omega},
$$

where $\bar{\beta}>0$ is the inf-sup condition constant for $\bar{W}_{H} \times \overline{\mathbf{V}}_{H}$.

To obtain a unique solution of the discrete approximation (2.31)-(2.32) of (1.4)(1.5), we can now apply the abstract inf-sup theory given in [16], for example. We can also obtain a bound on the approximation error, but it involves the approximation of $p$ in $\bar{W}_{H}$, which is only first order accurate. This is acceptable for RT0, but suboptimal for the higher order spaces.

THEOREM 4.4. There exists a unique solution $\left(\mathbf{u}_{H}, \bar{p}_{H}\right) \in \mathbf{V}_{H, *} \times \bar{W}_{H}$ to (2.31)(2.32). Moreover, there exists $C>0$, independent of $\epsilon$ and $H$, such that if $(\mathbf{u}, p)$ is the solution of (1.4)-(1.5), then

$$
\begin{aligned}
& \nabla \cdot \mathbf{u}_{H}=\nabla \cdot \mathbf{u}=f \\
& \left\|\mathbf{u}-\mathbf{u}_{H}\right\|_{0, \Omega} \leq C\left\{\inf _{\mathbf{v}_{H} \in \mathbf{V}_{H, *}, \nabla \cdot \mathbf{v}_{H}=\nabla \cdot \mathbf{u}}\left\|\mathbf{u}-\mathbf{v}_{H}\right\|_{0, \Omega}\right. \\
& \left.+\sup _{0 \neq \psi_{H} \in \hat{\mathbf{V}}_{H, *}, \nabla \cdot \psi_{H}=0} \frac{\left|\left(\alpha \mathbf{u}-\mathbf{b}, \psi_{H}\right)\right|}{\left\|\psi_{H}\right\|_{0, \Omega}}\right\}, \\
& \left\|\bar{p}-\bar{p}_{H}\right\|_{0, \Omega} \leq C\left\{\left\|\mathbf{u}-\mathbf{u}_{H}\right\|_{0, \Omega}\right. \\
& \left.+\sup _{0 \neq \psi_{H} \in \hat{\mathbf{V}}_{H, *}} \frac{\left|\left(\alpha \mathbf{u}-\mathbf{b}, \psi_{H}\right)-\sum_{E \in \mathcal{T}_{H}}\left(p, \nabla \cdot \psi_{H}\right)_{E}\right|}{\left\|\psi_{H}\right\|_{X}}\right\} .
\end{aligned}
$$

Proof. The first equality follows from (2.31). The inf-sup condition of the previous lemma and (2.32) allow us to estimate directly that

$$
\begin{aligned}
& \beta\left\|\bar{p}-\bar{p}_{H}\right\|_{0, \Omega} \\
& \leq \sup _{0 \neq \psi_{H} \in \hat{\mathbf{V}}_{H, *}} \frac{\sum_{E \in \mathcal{T}_{H}}\left(\bar{p}-\bar{p}_{H}, \nabla \cdot \psi_{H}\right)_{E}}{\left\|\psi_{H}\right\|_{X}} \\
& =\sup _{0 \neq \psi_{H} \in \hat{\mathbf{V}}_{H, *}} \frac{\sum_{E \in \mathcal{T}_{H}}\left[\left(p, \nabla \cdot \psi_{H}\right)_{E}-\left(\alpha \mathbf{u}_{H}-\mathbf{b}, \psi_{H}\right)_{E}\right]}{\left\|\psi_{H}\right\|_{X}} \\
& \leq \sup _{0 \neq \psi_{H} \in \hat{\mathbf{V}}_{H, *}} \frac{\sum_{E \in \mathcal{T}_{H}}\left[\left(p, \nabla \cdot \psi_{H}\right)_{E}-\left(\alpha \mathbf{u}-\mathbf{b}, \psi_{H}\right)_{E}\right]}{\left\|\psi_{H}\right\|_{X}}+\left\|\alpha\left(\mathbf{u}-\mathbf{u}_{H}\right)\right\|_{0, \Omega},
\end{aligned}
$$

and the third result (4.3) follows. 
The statement $\mathbf{v}_{H} \in \mathbf{V}_{H, *}$ such that $\nabla \cdot \mathbf{v}_{H}=\nabla \cdot \mathbf{u}=\nabla \cdot \mathbf{u}_{H}$ merely says that $\mathbf{v}_{H}-\mathbf{u}_{H} \in \hat{\mathbf{V}}_{H, *}$ and has vanishing divergence. For any such $\mathbf{v}_{H}$, we compute

$$
\begin{aligned}
\left\|\mathbf{u}-\mathbf{u}_{H}\right\|_{0, \Omega} \leq & \left\|\mathbf{u}-\mathbf{v}_{H}\right\|_{0, \Omega}+\left\|\mathbf{v}_{H}-\mathbf{u}_{H}\right\|_{0, \Omega} \\
\leq & \left\|\mathbf{u}-\mathbf{v}_{H}\right\|_{0, \Omega}+C \sup _{0 \neq \psi_{H} \in \hat{\mathbf{V}}_{H, *}, \nabla \cdot \psi_{H}=0} \frac{\left|\left(\alpha\left(\mathbf{v}_{H}-\mathbf{u}_{H}\right), \psi_{H}\right)\right|}{\left\|\psi_{H}\right\|_{0, \Omega}} \\
\leq & \left\|\mathbf{u}-\mathbf{v}_{H}\right\|_{0, \Omega}+C\left\{\sup _{0 \neq \psi_{H} \in \hat{\mathbf{V}}_{H, *}, \nabla \cdot \psi_{H}=0} \frac{\left|\left(\alpha\left(\mathbf{u}-\mathbf{u}_{H}\right), \psi_{H}\right)\right|}{\left\|\psi_{H}\right\|_{0, \Omega}}\right. \\
& \left.+\sup _{0 \neq \psi_{H} \in \hat{\mathbf{V}}_{H, *}, \nabla \cdot \psi_{H}=0} \frac{\left|\left(\alpha\left(\mathbf{v}_{H}-\mathbf{u}\right), \psi_{H}\right)\right|}{\left\|\psi_{H}\right\|_{0, \Omega}}\right\} \\
\leq & C\left\{\left\|\mathbf{u}-\mathbf{v}_{H}\right\|_{0, \Omega}+\sup _{0 \neq \psi_{H} \in \hat{\mathbf{V}}_{H, *}, \nabla \cdot \psi_{H}=0} \frac{\left|\left(\alpha \mathbf{u}-\mathbf{b}, \psi_{H}\right)\right|}{\left\|\psi_{H}\right\|_{0, \Omega}}\right\},
\end{aligned}
$$

since $\left(\alpha \mathbf{u}_{H}, \psi_{H}\right)=\left(\mathbf{b}, \psi_{H}\right)$, and the second result (4.4) follows.

Finally, we obtain that the discrete solution must be unique by setting all the data to zero $\left(f, \mathbf{b}, \mathbf{v}_{g}\right.$, which implies that $\mathbf{u}$ and $p$ also vanish). We then also obtain existence of a solution, since the system has finite dimensions and is square.

5. Some homogenization theory needed for multiscale error analysis. We give a multiscale analysis of the error similar to that given by Hou et al. [22, 17]. This analysis determines the behavior of the error as a function both of $H$ and the scale of the heterogeneity in $a$, which we denote by $\epsilon$. If $H \sim \epsilon$, the system is well resolved, there is no need for oversampling, and the scheme converges with optimal order of approximation [5]. Thus we tacitly assume the underresolved case where $\epsilon \ll H$. The difficulty, then, with standard approximation theory is that the error is bounded in terms of $H$ and derivatives of the solution. However, we expect that each derivative of the solution is proportional to $\epsilon^{-1}$, and $H / \epsilon$ is not small. The two exceptions are given by the standard energy estimates for our problem, which are stated in the following lemma.

Lemma 5.1. Let $(\mathbf{u}, p) \in\left(\mathbf{V}+\mathbf{v}_{g}\right) \times W$ be the solution of (1.4)-(1.5). Then there is a constant $C>0$, depending only on the ellipticity bounds for a, such that

$$
\begin{aligned}
& \|\mathbf{u}\|_{0, \Omega}+\|\nabla p\|_{0, \Omega} \leq C\left\{\left\|f-\nabla \cdot \mathbf{v}_{g}\right\|_{0, \Omega}+\left\|\mathbf{v}_{g}\right\|_{0, \Omega}+\|\mathbf{b}\|_{0, \Omega}\right\}, \\
& \|\nabla \cdot \mathbf{u}\|_{0, \Omega}=\|f\|_{0, \Omega} .
\end{aligned}
$$

In order to quantify the scale of the heterogeneity, we use homogenization theory. Thus we assume that the permeability has "locally periodic" oscillations whose scale is on the order of some $\epsilon>0$. That is, let $C_{\text {per }}^{1}\left(\mathbb{R}^{d}\right)$ denote the space of all $C^{1}\left(\mathbb{R}^{d}\right)$ functions that are periodic with respect to the unit cube $Y \subseteq \mathbb{R}^{d}$, and assume that $a=a(x, x / \epsilon)$, where for each $i, j=1, \ldots, d, a_{i j}(x, y) \in C^{1}\left(\bar{\Omega} ; C_{\mathrm{per}}^{1}\left(\mathbb{R}^{d}\right)\right)$, and $a$ varies slowly in its first argument on a scale resolved by $H$. Moreover, suppose $\mathbf{B} \in\left(L^{2}(D)\right)^{d}$.

Following Chen and Hou [17], we now review the relevant aspects of homogenization theory. Let $D \subseteq \Omega$ be a Lipschitz domain in $\mathbb{R}^{d}$ and suppose $F \in L^{2}(D)$ and $G \in L^{2}(\partial D)$ satisfy the compatibility condition

$$
\int_{D} F(x) d x=\int_{\partial D} G(x) d s
$$


For each $\epsilon>0$, we let $q^{\epsilon} \in H^{1}(D) / \mathbb{R}$ be the unique solution of the Neumann problem

$$
\int_{D} a(x, x / \epsilon)\left(\nabla q^{\epsilon}-\mathbf{B}\right) \cdot \nabla \varphi d x=\int_{D} F \varphi d x-\int_{\partial D} G \varphi d s \quad \forall \varphi \in H^{1}(D) .
$$

The homogenized coefficient matrix $a^{0}(x)=\left(a_{i j}^{0}(x)\right)_{i j}$ of $a(x, x / \epsilon)$ is given by

$$
a_{i j}^{0}(x)=\frac{1}{|Y|} \sum_{k=1}^{d} \int_{Y} a_{i k}(x, y)\left(\delta_{k j}-\frac{\partial \chi^{j}}{\partial y_{k}}(x, y)\right) d y, \quad x \in \Omega, y \in Y,
$$

where $\delta_{k j}$ is the Kronecker delta and $\chi^{j}(x, y)$ is the $Y$-periodic (in $y$ ) solution of the $j$ th cell problem

$$
\sum_{i=1}^{d} \sum_{k=1}^{d} \frac{\partial}{\partial y_{i}}\left(a_{i k}(x, y) \frac{\partial \chi^{j}}{\partial y_{k}}(x, y)\right)=\sum_{i=1}^{d} \frac{\partial}{\partial y_{i}} a_{i j}(x, y),
$$

with $\int_{Y} \chi^{j}(x, y) d y=0$. Now, we let $q^{0} \in H^{1}(D) / \mathbb{R}$ be the unique solution of the homogenized counterpart of (5.1), namely,

$$
\int_{D} a^{0}(x)\left(\nabla q^{0}-\mathbf{B}\right) \cdot \nabla \varphi d x=\int_{D} F \varphi d x-\int_{\partial D} G \varphi d s \forall \varphi \in H^{1}(D) .
$$

In the usual way, we define the first order corrector of $q^{\epsilon}$ by

$$
\left(q^{0}\right)_{1}^{\epsilon}(x)=q^{0}(x)-\epsilon \sum_{k=1}^{d} \chi^{k}(x, x / \epsilon)\left(\frac{\partial q^{0}}{\partial x_{k}}-B_{k}\right) .
$$

Recall that we use the notation $\|\cdot\|_{j, p, S}$ for the norm of the Sobolev space $W^{j, p}(S)$, and simply $\|\cdot\|_{j, S}$ if $p=2$.

Theorem 5.2. Suppose that $q^{0} \in H^{2}(D) \cap W^{1, \infty}(D)$ and $D^{\prime} \subset D$. Let the fluxes be denoted by

$$
\mathbf{U}^{\epsilon}=-a_{\epsilon}\left(\nabla q^{\epsilon}-\mathbf{B}\right) \quad \text { and } \quad \mathbf{U}^{0}=-a^{0}\left(\nabla q^{0}-\mathbf{B}\right) .
$$

There exist a constant $C$, independent of $\epsilon$, the size of the domains $D$ and $D^{\prime}$, and the terms $\mathbf{B}, F$, and $G$, and there exists a boundary corrector $\theta_{\epsilon}^{S} \in H^{1}(S) / \mathbb{R}$, defined below for $S \subset D$ in (5.11)-(5.12) and (5.9)-(5.10), such that

$$
\begin{aligned}
& \left\|\nabla\left[q^{\epsilon}-\left(q^{0}\right)_{1}^{\epsilon}-\epsilon \theta_{\epsilon}^{D}\right]\right\|_{0, D} \leq C \epsilon\left\|\nabla q^{0}-\mathbf{B}\right\|_{1, D}, \\
& \left\|\mathbf{U}^{\epsilon}-\left(\mathbf{U}^{0}+\epsilon a_{\epsilon} \nabla \theta_{\epsilon}^{D^{\prime}}-\epsilon a_{\epsilon} \nabla \theta_{\epsilon}^{D}+\psi_{\text {Sol }}^{D^{\prime}}\right)\right\|_{0, D^{\prime}} \leq C \epsilon\left\|\nabla q^{0}-\mathbf{B}\right\|_{1, D},
\end{aligned}
$$

where $\psi_{\text {Sol }}^{D^{\prime}}$ is a solenoidal vector, i.e., $\nabla \cdot \psi_{\text {Sol }}^{D^{\prime}}=0$ and $\psi_{\text {Sol }}^{D^{\prime}} \cdot \nu^{D^{\prime}}=0$ on $\partial D^{\prime}$. Moreover,

$$
\begin{aligned}
& \left\|\epsilon \nabla \theta_{\epsilon}^{S}\right\|_{0, S} \leq C\left\{\epsilon\left\|\nabla q^{0}-\mathbf{B}\right\|_{1, S}+\sqrt{\epsilon|\partial S|}\left\|\nabla q^{0}-\mathbf{B}\right\|_{0, \infty, S}\right\}, \\
& \left\|\epsilon \nabla \theta_{\epsilon}^{S}\right\|_{0, S} \leq C\left(\epsilon+H_{S}^{-1}(\epsilon|\partial S|)^{1 / d-\eta}\right)\left\|\nabla q^{0}-\mathbf{B}\right\|_{1, S},
\end{aligned}
$$

where $S$ is $D$ or $D^{\prime}, H_{S}=\operatorname{diam}(S)$, and $\eta=0$ if $d=3$ and $\eta$ is any fixed positive number if $d=2$.

REMARK 5.1. In [17], it is conjectured, but not proven, that estimate (5.7) can be improved by replacing $\sqrt{\epsilon|\partial S|}$ by $\epsilon \sqrt{|S|} / H_{S}$ in the oversampled case, i.e., with $S$ 
replaced by $S^{\prime} \subset S$ on the left-hand side. If this turns out to be the case, the estimates we derive can be correspondingly improved.

In [25], (5.5) and (5.7) were derived in the case where the coefficient $a=a(x)$ is periodic and $\mathbf{B}=0$. In [17], the proof was elucidated and extended to the case in point, where $a=a(x, x / \epsilon)$ is locally periodic. The proof easily modifies to handle the extra term related to $\mathbf{B}$, and we reproduce it here in brief so that we can extract the estimates (5.6) and (5.8).

Proof. We use the Einstein summation convention for repeated indices, and the more concise notation $\partial_{j}=\partial / \partial x_{j}$, and $\partial_{j}^{x}=\partial / \partial x_{j}$ and $\partial_{j}^{y}=\partial / \partial y_{j}$ if we are dealing with a function of $(x, y)$. The key to the proof is to note that

$$
a_{i k}^{0}(x)-a_{\epsilon, i j}(x, y)\left(\delta_{j k}-\partial_{j}^{y} \chi^{k}(x, y)\right)=\partial_{j}^{y} A_{i j}^{k}(x, y),
$$

where $A_{i j}^{k}(x, y)$ is skew-symmetric for each $k[25$, p. 6$]$. Let us denote

$$
\gamma_{i}(x)=\partial_{j}\left\{A_{i j}^{k}(x, x / \epsilon)\left[\partial_{k} q^{0}(x)-B_{k}(x)\right]\right\},
$$

so that

$$
\partial_{j}^{y} A_{i j}^{k}(x, x / \epsilon)\left(\partial_{k} q^{0}-B_{k}\right)=\epsilon \gamma_{i}-\epsilon \partial_{j}^{x} A_{i j}^{k}\left(\partial_{k} q^{0}-B_{k}\right)-\epsilon A_{i j}^{k} \partial_{j}\left(\partial_{k} q^{0}-B_{k}\right) .
$$

After some manipulation

$$
-a_{\epsilon, i j}\left[\partial_{j}\left(q^{0}\right)_{1}^{\epsilon}-B_{j}\right]=-a_{i j}^{0}\left(\partial_{j} q^{0}-B_{j}\right)+\epsilon \gamma_{i}+\epsilon \psi_{1, i},
$$

where

$$
\begin{aligned}
\psi_{1, i}= & -\partial_{j}^{x} A_{i j}^{k}\left(\partial_{k} q^{0}-B_{k}\right)-A_{i j}^{k} \partial_{j}\left(\partial_{k} q^{0}-B_{k}\right) \\
& +a_{\epsilon, i j} \partial_{j}^{x} \chi^{k}\left(\partial_{k} q^{0}-B_{k}\right)+a_{\epsilon, i j} \chi^{k} \partial_{j}\left(\partial_{k} q^{0}-B_{k}\right) .
\end{aligned}
$$

Now we let $\theta_{\epsilon}^{S} \in H^{1}(S) / \mathbb{R}$ be defined by

$$
\begin{array}{ll}
\nabla \cdot\left(a_{\epsilon} \nabla \theta_{\epsilon}^{S}\right)=0 & \text { in } S, \\
a_{\epsilon} \nabla \theta_{\epsilon}^{S} \cdot \nu^{S}=\gamma \cdot \nu^{S} & \text { on } \partial S,
\end{array}
$$

so that $\psi_{\text {Sol }}^{S}=\epsilon\left(\gamma-a_{\epsilon} \nabla \theta_{\epsilon}^{S}\right)$ has the requisite properties and

$$
-a_{\epsilon}\left[\nabla\left(q^{0}\right)_{1}^{\epsilon}-\mathbf{B}\right]=-a^{0}\left(\nabla q^{0}-\mathbf{B}\right)+\epsilon a_{\epsilon} \nabla \theta_{\epsilon}^{S}+\psi_{\text {Sol }}^{S}+\epsilon \psi_{1} .
$$

It is now a simple consequence of the governing equations (5.1) and (5.3) and the properties of $\psi_{\text {Sol }}^{D}$ that

$$
\left(a_{\epsilon} \nabla\left[q_{\epsilon}-\left(q^{0}\right)_{1}^{\epsilon}-\epsilon \theta_{\epsilon}^{D}\right], \nabla \varphi\right)_{D}=\epsilon\left(\psi_{1}, \nabla \varphi\right)_{D},
$$

and the first result (5.5) follows easily. The second result (5.6) follows from (5.13) and the previous result.

To obtain bounds on the boundary corrector, we use a smooth cut-off function $\zeta_{\epsilon}(x) \in[0,1]$ with compact support that is one except near $\partial S$, where it tends to zero in a narrow region of width $\epsilon$ with gradient bounded by $C / \epsilon$. Now let

$$
\begin{aligned}
& \gamma_{I, i}(x)=\partial_{j}\left\{A_{i j}^{k}(x, x / \epsilon)\left[\partial_{k} q^{0}(x)-B_{k}(x)\right] \zeta_{\epsilon}(x)\right\}, \\
& \gamma_{B, i}(x)=\partial_{j}\left\{A_{i j}^{k}(x, x / \epsilon)\left[\partial_{k} q^{0}(x)-B_{k}(x)\right]\left[1-\zeta_{\epsilon}(x)\right]\right\}
\end{aligned}
$$


(i.e., $\left.\gamma=\gamma_{B}+\gamma_{I}\right)$, and note that (5.11)-(5.12) imply that

$$
\left\|\nabla \theta_{\epsilon}\right\|_{0, S} \leq C\left\|\gamma_{B}\right\|_{0, S},
$$

since $\gamma_{B}$ is divergence free. Finally,

$$
\begin{aligned}
\epsilon\left\|\gamma_{B, i}\right\|_{0, S}= & \epsilon\left\|\partial_{j}\left\{A_{i j}^{k}\left[\partial_{k} q^{0}-B_{k}\right]\left[1-\zeta_{\epsilon}\right]\right\}\right\|_{0, S} \\
\leq & \epsilon\left\|\partial_{j}^{x} A_{i j}^{k}\left[\partial_{k} q^{0}-B_{k}\right]\left[1-\zeta_{\epsilon}\right]\right\|_{0, S}+\epsilon\left\|A_{i j}^{k} \partial_{j}\left[\partial_{k} q^{0}-B_{k}\right]\left[1-\zeta_{\epsilon}\right]\right\|_{0, S} \\
& +\epsilon\left\|A_{i j}^{k}\left[\partial_{k} q^{0}-B_{k}\right] \partial_{j}\left[1-\zeta_{\epsilon}\right]\right\|_{0, S}+\left\|\partial_{j}^{y} A_{i j}^{k}\left[\partial_{k} q^{0}-B_{k}\right]\left[1-\zeta_{\epsilon}\right]\right\|_{0, S} \\
\leq & C\left\{\epsilon\left\|\nabla q^{0}-\mathbf{B}\right\|_{1, S}+\left\|\nabla q^{0}-\mathbf{B}\right\|_{0, S \epsilon}^{\zeta}\right\},
\end{aligned}
$$

where $S_{\epsilon}^{\zeta}$ is the support of $1-\zeta_{\epsilon}$. Since the measure of $S_{\epsilon}^{\zeta}$ is proportional to $\epsilon|\partial S|$, we have

$$
\left\|\nabla q^{0}-\mathbf{B}\right\|_{0, S_{\epsilon}^{\zeta}} \leq \sqrt{\left|S_{\epsilon}^{\zeta}\right|}\left\|\nabla q^{0}-\mathbf{B}\right\|_{0, \infty, S} \leq C \sqrt{\epsilon|\partial S|}\left\|\nabla q^{0}-\mathbf{B}\right\|_{0, \infty, S},
$$

and (5.7) follows. To show (5.8), we instead use Hölder's inequality with $r=d /(d-2)$ (or large but finite if $d=2$ ) and the Sobolev imbedding theorem to show that

$$
\begin{aligned}
\left\|\nabla q^{0}-\mathbf{B}\right\|_{0, S_{\epsilon}^{\zeta}} & \leq C(\epsilon|\partial S|)^{(r-1) / 2 r}\left\|\nabla q^{0}-\mathbf{B}\right\|_{0,2 r, S_{\epsilon}^{\zeta}} \\
& \leq C H_{S}^{-1}(\epsilon|\partial S|)^{1 / d-\eta}\left\|\nabla q^{0}-\mathbf{B}\right\|_{1, S},
\end{aligned}
$$

wherein $\eta=0$ if $d=3$ and $\eta>0$ if $d=2$ (the factor $H_{S}^{-1}$ comes from a scaling argument on the size of the domain $S$ ). The proof is complete.

We will apply Theorem 5.2 several times, with $D$ being one of $\Omega, E$, or $E_{*}$. Since these are convex polygonal domains, the hypothesis $q^{0} \in H^{2}(D) \cap W^{1, \infty}(D)$ will hold provided that, for some $r>d, F \in L^{r}(D)$ and $G=\mathbf{v}_{g} \cdot \nu^{D}$ on $\partial D$ for some $\mathbf{v}_{g} \in\left(W^{1, r}(D)\right)^{d}[17,20,26]$.

6. Multiscale estimation of the errors. In this section, we estimate the terms in the basic estimates of Theorem 4.4 for the velocity and pressure errors. We obtain the following estimates which isolate the dependence on both $H$ and $\epsilon$.

TheOREm 6.1. For each $\epsilon>0$, let $\left(\mathbf{u}^{\epsilon}, p^{\epsilon}\right) \in\left(\mathbf{V}+\mathbf{v}_{g}\right) \times W$ be the solution of (1.4)(1.5) with the coefficient $a_{\epsilon}=a(x, x / \epsilon)$ and $\alpha_{\epsilon}=a_{\epsilon}^{-1}$. Let $\left(\mathbf{u}^{0}, p^{0}\right) \in\left(\mathbf{V}+\mathbf{v}_{g}\right) \times W$ satisfy (1.4)-(1.5) with the homogenized coefficient $a^{0}$ defined by (5.2) in place of a, and $\alpha^{0}=\left(a^{0}\right)^{-1}$. For $H>0$, let $\left(\hat{\mathbf{u}}_{H}^{\epsilon}, \bar{p}_{H}^{\epsilon}\right) \in \hat{\mathbf{V}}_{H, *} \times \bar{W}_{H}$ be the solution of the discrete upscaled equation (2.26)-(2.27), and define $\mathbf{u}_{H}^{\epsilon}$ by (2.29).

(a) Oversampling. Assume that the partition $\mathcal{T}_{H}$ consists only of simplices and $\overline{\mathbf{V}}_{H}$ is RT0. Then

$$
\begin{aligned}
\| \mathbf{u}^{\epsilon}- & \mathbf{u}_{H}^{\epsilon}\left\|_{H(\mathrm{div} ; \Omega)}+\right\| p^{\epsilon}-\bar{p}_{H}^{\epsilon} \|_{0, \Omega} \\
\leq & C\left\{(\epsilon+\sqrt{\epsilon / H}+H)\left[\left\|f-\nabla \cdot \mathbf{v}_{g}\right\|_{0, \Omega}+\left\|\mathbf{v}_{g}\right\|_{0, \Omega}+\|\mathbf{b}\|_{0, \Omega}\right]\right. \\
& +(\epsilon / H)\left\|\nabla p^{0}\right\|_{0, \infty, \Omega}+\sqrt{\epsilon / H}\left\|\nabla p^{0}-\mathbf{b}\right\|_{0, \infty, \Omega} \\
& \left.+(\epsilon+H)\left[\left\|\nabla p^{0}\right\|_{1, \Omega}+\left\|\nabla p^{0}-\mathbf{b}\right\|_{1, \Omega}\right]+H\left\|\mathbf{u}^{0}-\mathbf{v}_{g}\right\|_{1, \Omega}\right\} .
\end{aligned}
$$

If the oversampling conjecture of Chen and Hou [17] holds (Remark 5.1), then $\sqrt{\epsilon / H}$ may be replaced by $\epsilon / H$ above. 
(b) Nonoversampling. Assume that oversampling is not used. Let $m=1$ when $\overline{\mathbf{V}}_{H}$ is $R T 0$ and $m=1$ or 2 when $\overline{\mathbf{V}}_{H}$ is BDM1 or BDDF1. Then

$$
\begin{aligned}
\| \mathbf{u}^{\epsilon}- & \mathbf{u}_{H}^{\epsilon}\left\|_{H(\operatorname{div} ; \Omega)}+\right\| \bar{p}^{\epsilon}-\bar{p}_{H}^{\epsilon} \|_{0, \Omega} \\
\leq & C\left\{\epsilon\left\|\nabla p^{0}-\mathbf{b}\right\|_{1, \Omega}+\sqrt{\epsilon / H}\left\|\nabla p^{0}-\mathbf{b}\right\|_{0, \infty, \Omega}\right. \\
& \left.+H^{m}\left(\left\|\mathbf{u}^{0}-\mathbf{v}_{g}\right\|_{m, \Omega}+\left\|f-\nabla \cdot \mathbf{v}_{g}\right\|_{m-1, \Omega}\right)\right\} .
\end{aligned}
$$

Moreover, with $\eta=0$, if $d=3$, and $\eta$ any fixed positive number, if $d=2$,

$$
\begin{aligned}
\left\|\mathbf{u}^{\epsilon}-\mathbf{u}_{H}^{\epsilon}\right\|_{H(\operatorname{div} ; \Omega)} \leq & C\left\{\left(\epsilon+(\epsilon / H)^{1 / d-\eta}\right)\left\|\nabla p^{0}-\mathbf{b}\right\|_{1, \Omega}\right. \\
& \left.+H^{m}\left(\left\|\mathbf{u}^{0}-\mathbf{v}_{g}\right\|_{m, \Omega}+\left\|f-\nabla \cdot \mathbf{v}_{g}\right\|_{m-1, \Omega}\right)\right\} .
\end{aligned}
$$

We remark that (a) is a small improvement over the result in [17, Theorem 2.2]. Assuming the more pessimistic but proven bound on the boundary corrector, and with $\mathbf{v}_{g}=\mathbf{b}=0$, this previous result is

$$
\begin{aligned}
\left\|\mathbf{u}^{\epsilon}-\mathbf{u}_{H}^{\epsilon}\right\|_{H(\operatorname{div} ; \Omega)}+\left\|p^{\epsilon}-\bar{p}_{H}^{\epsilon}\right\|_{0, \Omega} \\
\leq C\left\{(\epsilon+H)\left(\left\|p^{0}\right\|_{2, \Omega}+\|f\|_{1, \Omega}+\left\|\mathbf{u}^{0}\right\|_{H(\operatorname{div} ; \Omega)}\right)\right. \\
\left.\quad+\sqrt{\epsilon / H}\left(\left\|p^{0}\right\|_{1, \infty, \Omega}+\|f\|_{0, \Omega}+\left\|\mathbf{u}^{0}\right\|_{H(\operatorname{div} ; \Omega)}\right)\right\} .
\end{aligned}
$$

The small improvement is in the norm on $f$, which as noted in the introduction can have small scale aspects in some applications such as flow in porous media. Result (b) is new for the BDM1 and BDDF1 spaces, and for RT0 with nonsimplicial elements.

Concerning the proof of this theorem, by Theorem 4.4, for (b), we need only to bound the optimal velocity error, which is done in section 6.1. For (a), we need this, the oversampling error, handled in section 6.2 , and the following simple estimate for the pressure. Note that in (6.1), we have $p^{\epsilon}$ rather than $\bar{p}^{\epsilon}$. This is allowed by the estimate

$$
\left\|p-\bar{p}_{H}\right\|_{0, \Omega} \leq\|p-\bar{p}\|_{0, \Omega}+\left\|\bar{p}-\bar{p}_{H}\right\|_{0, \Omega} \leq C H\|\nabla p\|_{0, \Omega}+\left\|\bar{p}-\bar{p}_{H}\right\|_{0, \Omega}
$$

and the bound on $\|\nabla p\|_{0, \Omega}$ in Lemma 5.1. We will improve the pressure estimate of (b) in section 7 .

6.1. The optimal velocity error. In this subsection, we assume that oversampling may be used, so as to handle cases (a) and (b) of Theorem 6.1 simultaneously. Let $\bar{\pi}_{H}: \mathbf{V} \cap L^{r}(\Omega) \rightarrow \overline{\mathbf{V}}_{H}$ (for some $r>2$ ) be the standard mixed finite element interpolation operator $[27,18,15,14,16]$. It has the property that

$$
\nabla \cdot \bar{\pi}_{H} \mathbf{v}=\mathcal{P}_{\bar{W}_{H}} \nabla \cdot \mathbf{v}
$$

for all $\mathbf{v} \in \mathbf{V} \cap L^{r}(\Omega)$, where $\mathcal{P}_{\bar{W}_{H}}$ is the $L^{2}$-projection onto $\bar{W}_{H}$. We also have the approximation property

$$
\left\|\mathbf{v}-\bar{\pi}_{H} \mathbf{v}\right\|_{0, \Omega} \leq C H^{m}\|\mathbf{v}\|_{m, \Omega},
$$

where $m=1$ when $\overline{\mathbf{V}}_{H}$ is RT0 and $m=1$ or 2 when $\overline{\mathbf{V}}_{H}$ is BDM1 or BDDF1.

We now note a lemma on the difference between nonoversampled and oversampled quantities.

Lemma 6.2. If $E \in \mathcal{T}_{H}$ and $w \in H^{1}\left(E_{*}\right)$, then

$$
\nabla \hat{p}_{*}^{\prime}(a \nabla w)=-\nabla w \quad \text { and } \quad \hat{\mathbf{u}}_{*}^{\prime}(a \nabla w)=0 .
$$


In fact, $\hat{p}_{*}^{\prime}(a \nabla w)=-w$ provided $w \in W_{*}^{\prime}\left(E_{*}\right)$. Moreover, on $E$,

$$
\nabla \hat{p}^{\prime}(a \nabla w)=\left.\nabla \hat{p}_{*}^{\prime}(a \nabla w)\right|_{E}=-\nabla w .
$$

Proof. We simply observe that (6.6) provides the unique solution to the equations defining the subgrid operator (2.20)-(2.21). The remark for $w \in W_{*}^{\prime}\left(E_{*}\right) \cap H^{1}\left(E_{*}\right)$ is then trivial, since $w$ is correctly normalized. Similar results hold for $\hat{p}^{\prime}$, so (6.7) follows.

Our main result in this subsection follows.

Lemma 6.3. Let

$$
\begin{aligned}
& \hat{\mathbf{v}}_{H}^{\epsilon}=\bar{\pi}_{H}\left(\mathbf{u}^{0}-\mathbf{v}_{g}\right)+\left.\sum_{E \in \mathcal{T}_{H}} \hat{\mathbf{u}}_{*}^{\prime}\left(\mathcal{E}_{E} \bar{\pi}_{H}\left(\mathbf{u}^{0}-\mathbf{v}_{g}\right)\right)\right|_{E} \in \hat{\mathbf{V}}_{H, *}, \\
& \mathbf{v}_{H}^{\epsilon}=\hat{\mathbf{v}}_{H}^{\epsilon}+\left.\sum_{E \in \mathcal{T}_{H}} \tilde{\mathbf{u}}_{*}^{\prime}\right|_{E}+\mathbf{v}_{g} \in \mathbf{V}_{H, *} .
\end{aligned}
$$

Then there is $C>0$, independent of $\epsilon$ and $H$, such that

$$
\begin{aligned}
\nabla \cdot \mathbf{u}^{\epsilon}= & \nabla \cdot \mathbf{v}_{H}^{\epsilon}, \\
\left\|\mathbf{u}^{\epsilon}-\mathbf{v}_{H}^{\epsilon}\right\|_{0, \Omega} \leq & C\left\{\epsilon\left\|\nabla p^{0}-\mathbf{b}\right\|_{1, \Omega}+\sqrt{\epsilon / H}\left\|\nabla p^{0}-\mathbf{b}\right\|_{0, \infty, \Omega}\right. \\
& \left.+H^{m}\left(\left\|\mathbf{u}^{0}-\mathbf{v}_{g}\right\|_{m, \Omega}+\left\|f-\nabla \cdot \mathbf{v}_{g}\right\|_{m-1, \Omega}\right)\right\}, \\
\left\|\mathbf{u}^{\epsilon}-\mathbf{v}_{H}^{\epsilon}\right\|_{0, \Omega} \leq & C\left\{\left(\epsilon+(\epsilon / H)^{1 / d-\eta}\right)\left\|\nabla p^{0}-\mathbf{b}\right\|_{1, \Omega}\right. \\
& \left.+H^{m}\left(\left\|\mathbf{u}^{0}-\mathbf{v}_{g}\right\|_{m, \Omega}+\left\|f-\nabla \cdot \mathbf{v}_{g}\right\|_{m-1, \Omega}\right)\right\},
\end{aligned}
$$

where $m$ is 1 or 2 and $\eta \geq 0$ as in Theorem 6.1.

Proof. The divergence result is easy to see from (6.4). For the other result, we work locally on $E_{*} \supset E \in \mathcal{T}_{H}$. We have an expansion over $E_{*}$ similar to the one over $E$, so on $E_{*}$ we can write

$$
p^{\epsilon}=\bar{p}_{*}+\hat{p}_{*}^{\prime}+\tilde{p}_{*}^{\prime},
$$

where $\bar{p}_{*}$ is the average of $p^{\epsilon}$ over $E_{*}$ and $\hat{p}_{*}^{\prime}, \tilde{p}_{*}^{\prime} \in W_{*}^{\prime}\left(E_{*}\right)$ are defined in (2.20)-(2.23) above. (To see this fact, simply consider an expansion as in section 2 on a perturbed coarse mesh containing $E_{*}$, and discard the expansion outside $E_{*}$.) In fact, we have $\mathbf{u}^{\epsilon}=\overline{\mathbf{u}}_{*}+\hat{\mathbf{u}}_{*}^{\prime}+\tilde{\mathbf{u}}_{*}^{\prime}+\mathbf{v}_{g}$ and the functional relationship

$$
\hat{p}_{*}^{\prime}=\hat{p}_{*}^{\prime}\left(\overline{\mathbf{u}}_{*}\right)=\hat{p}_{*}^{\prime}\left(\mathbf{u}^{\epsilon}-\tilde{\mathbf{u}}_{*}^{\prime}-\mathbf{v}_{g}\right),
$$

using Lemma 4.1 to avoid further discussion of $\overline{\mathbf{u}}_{*}$ and $\hat{\mathbf{u}}_{*}^{\prime}$. Thus we have on $E$ that

$$
\begin{aligned}
\mathbf{u}^{\epsilon} & =-a_{\epsilon}\left(\nabla p^{\epsilon}-\mathbf{b}\right) \\
& =-a_{\epsilon} \nabla \hat{p}_{*}^{\prime}\left(\mathbf{u}^{\epsilon}-\tilde{\mathbf{u}}_{*}^{\prime}-\mathbf{v}_{g}\right)-a_{\epsilon}\left(\nabla \tilde{p}_{*}^{\prime}-\mathbf{b}\right) \\
& =-a_{\epsilon}\left[\nabla \hat{p}_{*}^{\prime}\left(\mathbf{u}^{\epsilon}-\mathbf{u}^{0}\right)+\nabla \hat{p}_{*}^{\prime}\left(\mathbf{u}^{0}-\mathbf{v}_{g}\right)-\nabla \hat{p}_{*}^{\prime}\left(\tilde{\mathbf{u}}_{*}^{\prime}\right)\right]+\tilde{\mathbf{u}}_{*}^{\prime}+\mathbf{v}_{g},
\end{aligned}
$$

using (2.23) in the last step.

Note that on $E$,

$$
\left.\hat{\mathbf{v}}_{H}^{\epsilon}\right|_{E}=\bar{\pi}_{H}\left(\mathbf{u}^{0}-\mathbf{v}_{g}\right)+\left.\hat{\mathbf{u}}_{*}^{\prime}\left(\mathcal{E}_{E} \bar{\pi}_{H}\left(\mathbf{u}^{0}-\mathbf{v}_{g}\right)\right)\right|_{E}=-\left.a_{\epsilon} \nabla \hat{p}_{*}^{\prime}\left(\mathcal{E}_{E} \bar{\pi}_{H}\left(\mathbf{u}^{0}-\mathbf{v}_{g}\right)\right)\right|_{E},
$$


SO

$$
\begin{aligned}
\mathbf{u}^{\epsilon}-\mathbf{v}_{H}^{\epsilon} & =\mathbf{u}^{\epsilon}-\left(\hat{\mathbf{v}}_{H}^{\epsilon}+\tilde{\mathbf{u}}_{*}^{\prime}+\mathbf{v}_{g}\right) \\
& =-a_{\epsilon}\left[\nabla \hat{p}_{*}^{\prime}\left(\mathbf{u}^{\epsilon}-\mathbf{u}^{0}\right)+\nabla \hat{p}_{*}^{\prime}\left(\mathbf{u}^{0}-\mathbf{v}_{g}\right)-\nabla \hat{p}_{*}^{\prime}\left(\tilde{\mathbf{u}}_{*}^{\prime}\right)-\nabla \hat{p}_{*}^{\prime}\left(\mathcal{E}_{E} \bar{\pi}_{H}\left(\mathbf{u}^{0}-\mathbf{v}_{g}\right)\right)\right] .
\end{aligned}
$$

Now we estimate

$$
\begin{aligned}
\left\|\mathbf{u}^{\epsilon}-\mathbf{v}_{H}^{\epsilon}\right\|_{0, E} \leq & C\left\{\left\|\nabla \hat{p}_{*}^{\prime}\left(\mathbf{u}^{\epsilon}-\mathbf{u}^{0}\right)\right\|_{0, E}+\left\|\mathbf{u}^{0}-\mathbf{v}_{g}-\mathcal{E}_{E} \bar{\pi}_{H}\left(\mathbf{u}^{0}-\mathbf{v}_{g}\right)\right\|_{0, E_{*}}\right. \\
& \left.+\left\|\nabla \hat{p}_{*}^{\prime}\left(\tilde{\mathbf{u}}_{*}^{\prime}\right)\right\|_{0, E}\right\},
\end{aligned}
$$

using Lemma 4.1 again, this time to bound the operator. The second term on the right is bounded as

$$
\left\|\mathbf{u}^{0}-\mathbf{v}_{g}-\mathcal{E}_{E} \bar{\pi}_{H}\left(\mathbf{u}^{0}-\mathbf{v}_{g}\right)\right\|_{0, E_{*}} \leq C H^{m}\left\|\mathbf{u}^{0}-\mathbf{v}_{g}\right\|_{m, E_{*}},
$$

using the approximation property (6.5) of $\bar{\pi}_{H}$ (actually, a slight extension to $E_{*}$, but the approximation result continues to hold since the operator $\mathcal{E}_{E} \bar{\pi}_{H}$ preserves low order polynomials).

For the last term on the far right side of (6.9), since $\tilde{\mathbf{u}}_{*}^{\prime} \in \mathbf{V}_{*}^{\prime}\left(E_{*}\right)$, note that we have the differential system

$$
\begin{array}{ll}
-\nabla \cdot a_{\epsilon} \nabla \hat{p}_{*}^{\prime}\left(\tilde{\mathbf{u}}_{*}^{\prime}\right)=\mathcal{P}_{W_{*}^{\prime}}\left(f-\nabla \cdot \mathbf{v}_{g}\right) & \text { in } E_{*}, \\
-a_{\epsilon} \nabla \hat{p}_{*}^{\prime}\left(\tilde{\mathbf{u}}_{*}^{\prime}\right) \cdot \nu^{E_{*}}=0 & \text { on } \partial E_{*},
\end{array}
$$

where $\mathcal{P}_{W_{*}^{\prime}}$ is the $L^{2}$-projection onto $W_{*}^{\prime}\left(E_{*}\right)$. The standard energy estimate is

$$
\left\|\nabla \hat{p}_{*}^{\prime}\left(\tilde{\mathbf{u}}_{*}^{\prime}\right)\right\|_{0, E_{*}} \leq C\left\|\mathcal{P}_{W_{*}^{\prime}}\left(f-\nabla \cdot \mathbf{v}_{g}\right)\right\|_{\left(H^{1}\left(E_{*}\right)\right)^{*}} \leq C H^{m}\left\|f-\nabla \cdot \mathbf{v}_{g}\right\|_{m-1, E_{*}},
$$

where $\left(H^{1}\left(E_{*}\right)\right)^{*}$ is the dual space of $H^{1}\left(E_{*}\right)$, using standard negative norm estimates for approximation of a function with vanishing average.

Finally, we estimate the first term on the far right side of (6.9), using Theorem 5.2, specifically the expansion in (5.6). By Lemma 4.1 we can introduce the local solenoidal term $\psi_{\text {Sol }}^{E_{*}}$, so we have

$$
\begin{aligned}
& \nabla \hat{p}_{*}^{\prime}\left(\mathbf{u}^{\epsilon}-\mathbf{u}^{0}\right) \\
& \quad=\nabla \hat{p}_{*}^{\prime}\left(\mathbf{u}^{\epsilon}-\mathbf{u}^{0}-\epsilon a_{\epsilon} \nabla \theta_{\epsilon}^{E_{*}}+\epsilon a_{\epsilon} \nabla \theta_{\epsilon}^{\Omega}\right)+\epsilon \nabla \hat{p}_{*}^{\prime}\left(a_{\epsilon} \nabla \theta_{\epsilon}^{E_{*}}\right)-\epsilon \nabla \hat{p}_{*}^{\prime}\left(a_{\epsilon} \nabla \theta_{\epsilon}^{\Omega}\right) \\
& \quad=\nabla \hat{p}_{*}^{\prime}\left(\mathbf{u}^{\epsilon}-\mathbf{u}^{0}-\epsilon a_{\epsilon} \nabla \theta_{\epsilon}^{E_{*}}+\epsilon a_{\epsilon} \nabla \theta_{\epsilon}^{\Omega}+\psi_{\text {Sol }}^{E_{*}}\right)-\epsilon \nabla \theta_{\epsilon}^{E_{*}}+\epsilon \nabla \theta_{\epsilon}^{\Omega},
\end{aligned}
$$

using Lemma 6.2. Thus Theorem 5.2 gives the two bounds

$$
\begin{aligned}
& \left\|\nabla \hat{p}_{*}^{\prime}\left(\mathbf{u}^{\epsilon}-\mathbf{u}^{0}\right)\right\|_{0, E} \\
& \quad \leq C\left\{\epsilon\left\|\nabla p^{0}-\mathbf{b}\right\|_{1, E_{*}}+\sqrt{\epsilon\left|\partial E_{*}\right|}\left\|\nabla p^{0}-\mathbf{b}\right\|_{0, \infty, E_{*}}+\left\|\epsilon \nabla \theta_{\epsilon}^{\Omega}\right\|_{0, E_{*}}\right\} \\
& \left\|\nabla \hat{p}_{*}^{\prime}\left(\mathbf{u}^{\epsilon}-\mathbf{u}^{0}\right)\right\|_{0, E} \leq C\left\{\left(\epsilon+H^{-1}\left(\epsilon\left|\partial E_{*}\right|\right)^{1 / d-\eta}\right)\left\|\nabla p^{0}-\mathbf{b}\right\|_{1, E_{*}}+\left\|\epsilon \nabla \theta_{\epsilon}^{\Omega}\right\|_{0, E_{*}}\right\}
\end{aligned}
$$

(with the first bound improved if the oversampling conjecture holds).

Combining terms, summing over $E \in \mathcal{T}_{H}$, and using that the number of overlaps of the $E_{*}$ are bounded yield

$$
\begin{aligned}
\left\|\mathbf{u}^{\epsilon}-\mathbf{v}_{H}^{\epsilon}\right\|_{0, \Omega} \leq & C\left\{\epsilon\left\|\nabla p^{0}-\mathbf{b}\right\|_{1, \Omega}+\sqrt{\epsilon / H}\left\|\nabla p^{0}-\mathbf{b}\right\|_{0, \infty, \Omega}\right. \\
& \left.+\left\|\epsilon \nabla \theta_{\epsilon}^{\Omega}\right\|_{0, \Omega}+H^{m}\left(\left\|\mathbf{u}^{0}-\mathbf{v}_{g}\right\|_{m, \Omega}+\left\|f-\nabla \cdot \mathbf{v}_{g}\right\|_{m-1, \Omega}\right)\right\},
\end{aligned}
$$


wherein $\sqrt{\epsilon / H}\left\|\nabla p^{0}-\mathbf{b}\right\|_{0, \infty, \Omega}$ can be replaced by $(\epsilon / H)^{1 / d-\eta}\left\|\nabla p^{0}-\mathbf{b}\right\|_{1, \Omega}$. The proof is completed by Theorem 5.2 to bound the global boundary corrector term.

We have an abstract result, analogous to Theorem 5.2, relating $\mathbf{u}^{\epsilon}$ to a correction of the homogenized solution $\mathbf{u}^{0}$.

Corollary 6.4. If $\tilde{\mathbf{u}}_{*}^{\prime}\left(\mathbf{u}^{0}\right)$ is defined by (2.22)-(2.23) with $\mathbf{v}_{g}$ replaced by $\mathbf{u}^{0}$, then

$$
\begin{aligned}
& \left\|\mathbf{u}^{\epsilon}-\left(\mathbf{u}^{0}+\left.\sum_{E \in \mathcal{T}_{H}} \tilde{\mathbf{u}}_{*}^{\prime}\left(\mathbf{u}^{0}\right)\right|_{E}\right)\right\|_{0, \Omega} \leq C\left\{\epsilon\left\|\nabla p^{0}-\mathbf{b}\right\|_{1, \Omega}+\sqrt{\epsilon / H}\left\|\nabla p^{0}-\mathbf{b}\right\|_{0, \infty, \Omega}\right\}, \\
& \left\|\mathbf{u}^{\epsilon}-\left(\mathbf{u}^{0}+\left.\sum_{E \in \mathcal{T}_{H}} \tilde{\mathbf{u}}_{*}^{\prime}\left(\mathbf{u}^{0}\right)\right|_{E}\right)\right\|_{0, \Omega} \leq C\left(\epsilon+(\epsilon / H)^{1 / d-\eta}\right)\left\|\nabla p^{0}-\mathbf{b}\right\|_{1, \Omega} .
\end{aligned}
$$

Proof. Simply take $\mathbf{v}_{g}=\mathbf{u}^{0}$. Then $\hat{\mathbf{v}}_{H}^{\epsilon}=0$, and we can remove the term involving $f$ from the estimate since $\nabla \cdot \mathbf{v}_{g}=\nabla \cdot \mathbf{u}^{0}=f$.

6.2. The oversampled nonconforming error. Chen and Hou [17, pp. 559563] bounded the nonconforming error terms in Theorem 4.4 when $\mathbf{b}=0$. The key features needed in the analysis are (1) that the vector variable of the RT0 spaces, when restricted to an element and multiplied by a constant matrix, is a pure potential (i.e., a gradient of a scalar function), and (2) a vector variable $\overline{\mathbf{v}}_{H}$ in RT0 satisfies the estimate

$$
\left\|\overline{\mathbf{v}}_{H}\right\|_{1, E} \leq C\left\|\overline{\mathbf{v}}_{H}\right\|_{H(\operatorname{div} ; E)}
$$

(see $[17,(4.26)])$. These properties hold only for RT0 on simplices.

The extension of their result to nonzero $\mathbf{b}$ is not difficult, and, again using the more pessimistic but proven bound on the homogenization boundary corrector terms (see Remark 5.1), the extended result follows.

LEMma 6.5. There is a constant $C>0$, independent of $H$ and $\epsilon$, such that for any $\psi_{H} \in \hat{\mathbf{V}}_{H, *}$,

$$
\begin{aligned}
\mid(\alpha \mathbf{u} & \left.-\mathbf{b}, \psi_{H}\right)-\sum_{E \in \mathcal{T}_{H}}\left(p, \nabla \cdot \psi_{H}\right)_{E} \mid \\
\leq & C\left\{(\epsilon+\sqrt{\epsilon / H}+H)\left[\left\|f-\nabla \cdot \mathbf{v}_{g}\right\|_{0, \Omega}+\left\|\mathbf{v}_{g}\right\|_{0, \Omega}+\|\mathbf{b}\|_{0, \Omega}\right]\right. \\
& +(\epsilon+H)\left[\left\|\nabla p^{0}\right\|_{1, \Omega}+\left\|\nabla p^{0}-\mathbf{b}\right\|_{1, \Omega}\right] \\
& \left.+(\epsilon / H)\left\|\nabla p^{0}\right\|_{0, \infty, \Omega}+\sqrt{\epsilon / H}\left\|\nabla p^{0}-\mathbf{b}\right\|_{0, \infty, \Omega}\right\}\left\|\psi_{H}\right\|_{X} .
\end{aligned}
$$

This completes the proof of Theorem 6.1.

7. Superconvergent multiscale estimation of the pressure error. In this section, we assume that oversampling is not used. In this case, we can significantly improve the estimate of the pressure error over that obtained in Theorem 6.1 above.

TheOREm 7.1. For each $\epsilon>0$, let $\left(\mathbf{u}^{\epsilon}, p^{\epsilon}\right) \in\left(\mathbf{V}+\mathbf{v}_{g}\right) \times W$ be the solution of (1.4)-(1.5), with the coefficient $a_{\epsilon}=a(x, x / \epsilon)$ and $\alpha_{\epsilon}=a_{\epsilon}^{-1}$. For each $H>0$, let $\left(\hat{\mathbf{u}}_{H}^{\epsilon}, \bar{p}_{H}^{\epsilon}\right) \in \hat{\mathbf{V}}_{H} \times \bar{W}_{H}$ be the solution of the nonoversampled $\left(E_{*}=E \forall E \in \mathcal{T}_{H}\right)$ discrete upscaled equation (2.26)-(2.27), and define $\left(\mathbf{u}_{H}^{\epsilon}, p_{H}^{\epsilon}\right)$ by (2.29)-(2.30). Let $m=1$ when $\overline{\mathbf{V}}_{H}$ is the RT0 space and $m=1$ or 2 when $\overline{\mathbf{V}}_{H}$ is BDM1 or BDDF1. Assume that the domain $\Omega$ is $k$-regular, in the sense of (7.3) below. If $k=2$, then

$$
\left\|p^{\epsilon}-p_{H}^{\epsilon}\right\|_{0, \Omega} \leq C\left(\epsilon+(\epsilon / H)^{1 / d-\eta}+H\right)\left\|\mathbf{u}^{\epsilon}-\mathbf{u}_{H}^{\epsilon}\right\|_{0, \Omega},
$$


and if $k=3$, then

$$
\left\|p^{\epsilon}-p_{H}^{\epsilon}\right\|_{-1, \Omega} \leq C\left(\epsilon+(\epsilon / H)^{1 / d-\eta}+H^{m}\right)\left\|\mathbf{u}^{\epsilon}-\mathbf{u}_{H}^{\epsilon}\right\|_{0, \Omega} .
$$

These results display superconvergence, in that the pressure converges at a rate better that we would normally expect from approximation theory. Combining Theorems 7.1 and $6.1(\mathrm{~b})$, we obtain for $d=2$ that

$$
\left\|p^{\epsilon}-p_{H}^{\epsilon}\right\|_{0, \Omega} \leq C\left(\epsilon^{2}+\epsilon / H+H^{m+1}\right) .
$$

One should compare this estimate to the $L^{2}$-estimate of Efendiev, Hou, and Wu [19] for the (nonmixed) multiscale finite element method:

$$
\left\|p^{\epsilon}-p_{H}^{\epsilon}\right\|_{0, \Omega} \leq C\left(\epsilon+\epsilon|\ln h|+(\epsilon / H)^{2}+C_{\theta} \epsilon / H+H^{2}\right)
$$

although numerical results suggest that $C_{\theta}$ is negligible.

Proof. The difference between (1.5) and the conforming, nonoversampled method (2.32) is

$$
\left(\alpha\left(\mathbf{u}-\mathbf{u}_{H}\right), \hat{\mathbf{v}}_{H}\right)=\left(\bar{p}-\bar{p}_{H}, \nabla \cdot \overline{\mathbf{v}}_{H}\right) \quad \forall \hat{\mathbf{v}}_{H} \in \hat{\mathbf{V}}_{H}
$$

(wherein we suppress the superscript $\epsilon$ on the solutions and the subscript $\epsilon$ on $\alpha$ ). For $\varphi \in H^{k-2}(\Omega)$, we construct a test function from the solution $\mathbf{U} \in \mathbf{V}$ to the problem

$$
\begin{array}{ll}
\nabla \cdot \mathbf{U}=\varphi & \text { in } \Omega, \\
\mathbf{U}=-a \nabla q & \text { in } \Omega, \\
\mathbf{U} \cdot \nu=0 & \text { on } \partial \Omega .
\end{array}
$$

This is the same problem as (1.1)-(1.3), with $f=\varphi, \mathbf{b}=0$, and $g=0$ (i.e., $\mathbf{v}_{g}=0$ ). We solve this problem approximately with the variational multiscale method (2.31)(2.32) of section 2 for $\mathbf{U}_{H}=\hat{\mathbf{U}}_{H}+\tilde{\mathbf{U}}^{\prime} \in \hat{\mathbf{V}}_{H}+\tilde{\mathbf{U}}^{\prime}$. Note that $\nabla \cdot \hat{\mathbf{U}}_{H}=\bar{\varphi}, \tilde{\mathbf{U}}^{\prime}=-a \nabla \tilde{q}^{\prime}$, and Theorem 6.1 imply that

$$
\left\|\mathbf{U}-\mathbf{U}_{H}\right\|_{0, \Omega} \leq C\left\{\left(\epsilon+(\epsilon / H)^{1 / d-\eta}\right)\left\|\nabla q^{0}\right\|_{1, \Omega}+H^{m}\left(\left\|\mathbf{U}^{0}\right\|_{m, \Omega}+\|\varphi\|_{m-1, \Omega}\right)\right\},
$$

where $\left(\mathbf{U}^{0}, q^{0}\right) \in \mathbf{V} \times W$ satisfies the corresponding homogenized problem (i.e., with $a^{0}$ replacing $a$ ). The $k$-regularity assumption means that there is some constant $C>0$, independent of $H$ and $\epsilon$, such that

$$
\left\|\mathbf{U}^{0}\right\|_{k-1, \Omega}+\left\|q^{0}\right\|_{k, \Omega} \leq C\|\varphi\|_{k-2, \Omega},
$$

so

$$
\left\|\mathbf{U}-\mathbf{U}_{H}\right\|_{0, \Omega} \leq C\left(\epsilon+(\epsilon / H)^{1 / d-\eta}+H^{m}\right)\|\varphi\|_{k-2, \Omega},
$$

wherein $m=1$ if $k=2$.

Using the test function $\hat{\mathbf{U}}_{H} \in \hat{\mathbf{V}}_{H}$ in (7.2), we obtain that

$$
\begin{aligned}
& \left(\bar{p}-\bar{p}_{H}, \bar{\varphi}\right)=\left(\bar{p}-\bar{p}_{H}, \varphi\right)=\left(\alpha\left(\mathbf{u}-\mathbf{u}_{H}\right), \hat{\mathbf{U}}_{H}\right) \\
& \quad=\left(\alpha\left(\mathbf{u}-\mathbf{u}_{H}\right), \mathbf{U}_{H}-\mathbf{U}\right)+\left(\alpha\left(\mathbf{u}-\mathbf{u}_{H}\right), \mathbf{U}-\tilde{\mathbf{U}}^{\prime}\right) .
\end{aligned}
$$


Now, by the divergence theorem,

$$
\begin{aligned}
& \left(\alpha\left(\mathbf{u}-\mathbf{u}_{H}\right), \mathbf{U}-\tilde{\mathbf{U}}^{\prime}\right)=-\left(\alpha\left(\mathbf{u}-\mathbf{u}_{H}\right), a \nabla\left(q-\tilde{p}^{\prime}\right)\right) \\
& \quad=-\left(\mathbf{u}-\mathbf{u}_{H}, \nabla\left(q-\tilde{p}^{\prime}\right)\right)=\left(\nabla \cdot\left(\mathbf{u}-\mathbf{u}_{H}\right), q-\tilde{p}^{\prime}\right)=0
\end{aligned}
$$

so

$$
\begin{aligned}
\left(\bar{p}-\bar{p}_{H}, \varphi\right) & =\left(\alpha\left(\mathbf{u}-\mathbf{u}_{H}\right), \mathbf{U}_{H}-\mathbf{U}\right) \\
& \leq C\left\|\mathbf{u}-\mathbf{u}_{H}\right\|_{0, \Omega}\left\|\mathbf{U}-\mathbf{U}_{H}\right\|_{0, \Omega} \\
& \leq C\left\|\mathbf{u}-\mathbf{u}_{H}\right\|_{0, \Omega}\left(\epsilon+(\epsilon / H)^{1 / d-\eta}+H^{m}\right)\|\varphi\|_{k-2, \Omega}
\end{aligned}
$$

wherein $m=1$ if $k=2$.

Taking $k=2$ and the supremum over $\varphi \in L^{2}(\Omega)$, we see the estimate

$$
\left\|\bar{p}-\bar{p}_{H}\right\|_{0, \Omega} \leq C\left(\epsilon+(\epsilon / H)^{1 / d-\eta}+H\right)\left\|\mathbf{u}-\mathbf{u}_{H}\right\|_{0, \Omega} .
$$

If instead $k=3$ and $\varphi \in H_{0}^{1}(\Omega)$, we obtain

$$
\left\|\bar{p}-\bar{p}_{H}\right\|_{-1, \Omega} \leq C\left(\epsilon+(\epsilon / H)^{1 / d-\eta}+H^{m}\right)\left\|\mathbf{u}-\mathbf{u}_{H}\right\|_{0, \Omega} .
$$

Now by Lemma 4.1, we see that

$$
\hat{p}^{\prime}\left(\overline{\mathbf{u}}-\overline{\mathbf{u}}_{H}\right)=\hat{p}^{\prime}\left(\overline{\mathbf{u}}+\hat{\mathbf{u}}^{\prime}(\overline{\mathbf{u}})-\overline{\mathbf{u}}_{H}-\hat{\mathbf{u}}^{\prime}\left(\overline{\mathbf{u}}_{H}\right)\right)=\hat{p}^{\prime}\left(\mathbf{u}-\mathbf{u}_{H}\right)
$$

so we conclude that

$$
\begin{aligned}
\left\|p-p_{H}\right\|_{0, \Omega} & \leq\left\|\bar{p}-\bar{p}_{H}\right\|_{0, \Omega}+\left\|\hat{p}^{\prime}\left(\mathbf{u}-\mathbf{u}_{H}\right)\right\|_{0, \Omega} \\
& \leq\left\|\bar{p}-\bar{p}_{H}\right\|_{0, \Omega}+C H\left\|\nabla \hat{p}^{\prime}\left(\mathbf{u}-\mathbf{u}_{H}\right)\right\|_{0, \Omega} \\
& \leq\left\|\bar{p}-\bar{p}_{H}\right\|_{0, \Omega}+C H\left\|\mathbf{u}-\mathbf{u}_{H}\right\|_{0, \Omega} \\
& \leq C\left(\epsilon+(\epsilon / H)^{1 / d-\eta}+H\right)\left\|\mathbf{u}-\mathbf{u}_{H}\right\|_{0, \Omega},
\end{aligned}
$$

which is our first estimate. For the negative norm estimate,

$$
\left\|\hat{p}^{\prime}\left(\mathbf{u}-\mathbf{u}_{H}\right)\right\|_{-1, \Omega} \leq C H^{2}\left\|\nabla \hat{p}^{\prime}\left(\mathbf{u}-\mathbf{u}_{H}\right)\right\|_{0, \Omega} \leq C H^{2}\left\|\mathbf{u}-\mathbf{u}_{H}\right\|_{0, \Omega},
$$

so if $k=3$,

$$
\begin{aligned}
\left\|p-p_{H}\right\|_{-1, \Omega} & \leq\left\|\bar{p}-\bar{p}_{H}\right\|_{-1, \Omega}+\left\|\hat{p}^{\prime}\left(\mathbf{u}-\mathbf{u}_{H}\right)\right\|_{-1, \Omega} \\
& \leq C\left(\epsilon+(\epsilon / H)^{1 / d-\eta}+H^{m}\right)\left\|\mathbf{u}-\mathbf{u}_{H}\right\|_{0, \Omega}
\end{aligned}
$$

completing the proof.

\section{REFERENCES}

[1] J. E. AARnES, On the use of a mixed multiscale finite element method for greater flexibility and increased speed or improved accuracy in reservoir simulation, Multiscale Model. Simul., 2 (2004), pp. 421-439.

[2] J. E. Aarnes, S. Krogstad, And K.-A. Lie, A hierarchical multiscale method for two-phase flow based upon mixed finite elements and nonuniform coarse grids, Multiscale Model. Simul., 5 (2006), pp. 337-363.

[3] T. Arbogast, Numerical subgrid upscaling of two-phase flow in porous media, in Numerical Treatment of Multiphase Flows in Porous Media, Z. Chen, R. E. Ewing, and Z.-C. Shi, eds., Lecture Notes in Phys. 552, Springer-Verlag, Berlin, 2000, pp. 35-49. 
[4] T. Arbogast, Implementation of a locally conservative numerical subgrid upscaling scheme for two-phase Darcy flow, Comput. Geosci., 6 (2002), pp. 453-481.

[5] T. Arbogast, Analysis of a two-scale, locally conservative subgrid upscaling for elliptic problems, SIAM J. Numer. Anal., 42 (2004), pp. 576-598.

[6] T. Arbogast and S. L. Bryant, A two-scale numerical subgrid technique for waterflood simulations, SPE J., 7 (2002), pp. 446-457.

[7] T. Arbogast, S. E. Minkoff, and P. T. Keenan, An operator-based approach to upscaling the pressure equation, in Computational Methods in Water Resources XII, Vol. 1: Computational Methods in Contamination and Remediation of Water Resources, V. N. Burganos et al., eds., Computational Mechanics Publications, Southampton, U.K., 1998, pp. 405-412.

[8] I. BABUŠKA, The finite element method with Lagrangian multipliers, Numer. Math., 20 (1973), pp. 179-192.

[9] I. BabušKa, G. CAloz, And J. E. Osborn, Special finite element methods for a class of second order elliptic problems with rough coefficients, SIAM J. Numer. Anal., 31 (1994), pp. 945-981.

[10] I. BABUŠKA AND J. E. Osborn, Generalized finite element methods: Their performance and their relation to mixed methods, SIAM J. Numer. Anal., 20 (1983), pp. 510-536.

[11] S. C. Brenner And L. R. Scott, The Mathematical Theory of Finite Element Methods, Springer-Verlag, New York, 1994.

[12] F. BREZZI, On the existence, uniqueness and approximation of saddle-point problems arising from Lagrangian multipliers, Rev. Française Automat. Informat. Recherche Opérationelle Sér. Rouge, 8 (1974), pp. 129-151.

[13] F. BrezzI, Interacting with the subgrid world, in Numerical Analysis 1999, Chapman \& Hall/CRC, Boca Raton, FL, 2000, pp 69-82.

[14] F. Brezzi, J. Douglas, Jr., R. Duràn, and M. Fortin, Mixed finite elements for second order elliptic problems in three variables, Numer. Math., 51 (1987), pp. 237-250.

[15] F. Brezzi, J. Douglas, JR., And L. D. Marini, Two families of mixed elements for second order elliptic problems, Numer. Math., 47 (1985), pp. 217-235.

[16] F. Brezzi And M. Fortin, Mixed and Hybrid Finite Element Methods, Springer-Verlag, New York, 1991.

[17] Z. Chen And T. Y. Hou, A mixed multiscale finite element method for elliptic problems with oscillating coefficients, Math. Comp., 72 (2003), pp. 541-576.

[18] J. Douglas, JR. And J. E. Roberts, Global estimates for mixed methods for second order elliptic equations, Math. Comp., 44 (1985), pp. 39-52.

[19] Y. R. Efendiev, T. Y. Hou, AND X.-H. WU, Convergence of a nonconforming multiscale finite element method, SIAM J. Numer. Anal., 37 (2000), pp. 888-910.

[20] P. Grisvard, Elliptic Problems in Nonsmooth Domains, Pitman, Boston, 1985.

[21] T. Y. Hou AND X. H. Wu, A multiscale finite element method for elliptic problems in composite materials and porous media, J. Comput. Phys., 134 (1997), pp. 169-189.

[22] T. Y. Hou, X.-H. Wu, AND Z. CAI, Convergence of a multiscale finite element method for elliptic problems with rapidly oscillating coefficients, Math. Comp., 68 (1999), pp. 913943.

[23] T. J. R. Hughes, Multiscale phenomena: Green's functions, the Dirichlet-to-Neumann formulation, subgrid scale models, bubbles and the origins of stabilized methods, Comput. Methods Appl. Mech. Engrg., 127 (1995), pp. 387-401.

[24] T. J. R. Hughes, G. R. Feisóo, L. Mazzei, And J.-B. Quincy, The variational multiscale method - a paradigm for computational mechanics, Comput. Methods Appl. Mech. Engrg., 166 (1998), pp. 3-24.

[25] V. V. Jikov, S. M. Kozlov, And O. A. Oleinik, Homogenization of Differential Operators and Integral Functions, Springer-Verlag, New York, 1994.

[26] G. M. Lieberman, Oblique derivative problems in Lipschitz domains II. Discontinuous boundary data, J. Reine Angew. Math., 389 (1988), pp. 1-21.

[27] R. A. Raviart and J. M. Thomas, A mixed finite element method for 2 nd order elliptic problems, in Mathematical Aspects of Finite Element Methods, I. Galligani and E. Magenes, eds., Lecture Notes in Math. 606, Springer-Verlag, New York, 1977, pp. 292-315. 